CERN-TH/98-31

hep-ph/9801375

\title{
NLL Resummation \\ OF THE HEAVY-QUARK \\ Hadroproduction Cross-Section
}

\author{
Roberto BONCIANI, \\ INFN and Dipartimento di Fisica dell'Università, \\ Largo E. Fermi 2, Firenze, Italy
Stefano CATANI ${ }^{1}$, Michelangelo L. MANGANO ${ }^{2}$ and Paolo NASON ${ }^{3}$
CERN, Theoretical Physics Division,
CH 1211 Geneva 23, Switzerland

\begin{abstract}
We compute the effect of soft-gluon resummation, at the next-to-leading-logarithmic level, in the hadroproduction cross-section for heavy flavours. Applications to top, bottom and charm total cross-sections are discussed. We find in general that the corrections to the fixed next-to-leading-order results are larger for larger renormalization scales, and small, or even negative, for smaller scales. This leads to a significant reduction of the scale-dependence of the results, for most experimental configurations of interest.
\end{abstract}

CERN-TH/98-31

January 1998

\footnotetext{
${ }^{1}$ On leave of absence from INFN, Firenze, Italy

${ }^{2}$ On leave of absence from INFN, Pisa, Italy

${ }^{3}$ On leave of absence from INFN, Milano, Italy
} 


\section{Introduction}

Large logarithmically-enhanced corrections due to soft-gluon radiation are a general feature in the study of the production cross-sections of high-mass systems near threshold. Techniques for resumming these corrections have been developed over the past several years, starting from the case of Drell-Yan (DY) pair production $[1,2]$. In this paper we deal with the resummation of logarithmically-enhanced effects in the vicinity of the threshold region in the total cross-section for the hadroproduction of heavy quarks. This topic has been examined in several studies in recent years [3]-[7]. These analyses were limited to soft-gluon resummation to leading-logarithmic accuracy. The work we present here extends and updates the results of refs. [6] and [7] by consistently including all the next-to-leading logarithmic contributions.

The resummation program of soft-gluon contributions is best carried out in the Mellin-transform space, or $N$-space. In $N$-moment space the threshold-production region corresponds to the limit $N \rightarrow \infty$ and the typical structure of the threshold corrections is as follows

$$
\hat{\sigma}_{N}^{(L O)}\left\{1+\sum_{n=1}^{\infty} \alpha_{s}^{n} \sum_{m=1}^{2 n} c_{n, m} \ln ^{m} N\right\},
$$

where $\hat{\sigma}_{N}^{(L O)}$ is the corresponding partonic cross-section at leading order (LO). In the case of hadronic collisions, the best studied process $[1,2]$ is Drell-Yan (DY) lepton-pair production. In the DY process the logarithmic terms in the curly bracket of eq. (1) can be explicitly summed and organized in a radiative factor $\Delta_{D Y, N}$ that has an exponential form:

$$
\begin{aligned}
\Delta_{D Y, N}\left(\alpha_{s}\right) & =\exp \left\{\sum_{n=1}^{\infty} \alpha_{s}^{n} \sum_{m=1}^{n+1} G_{n m} \ln ^{m} N\right\} \\
& =\exp \left\{\ln N g_{D Y}^{(1)}\left(\alpha_{s} \ln N\right)+g_{D Y}^{(2)}\left(\alpha_{s} \ln N\right)+\alpha_{s} g_{D Y}^{(3)}\left(\alpha_{s} \ln N\right)+\ldots\right\} .
\end{aligned}
$$

Note that the exponentiation in eq. (2) is not trivial. The sum over $m$ in eq. (1) extends up to $m=2 n$ while in eq. (2) the maximum value for $m$ is smaller, $m \leq n+1$. In particular, this means that all the double logarithmic (DL) terms $\alpha_{s}^{n} c_{n, 2 n} \ln ^{2 n} N$ in eq. (1) are taken into account by simply exponentiating the lowest-order contribution $\alpha_{s} c_{1,2} \ln ^{2} N$. Then, the exponentiation in eq. (2) allows one to define the improved perturbative expansion in eq. (3). The function $\ln N g_{D Y}^{(1)}$ resums all the leading logarithmic (LL) contributions $\alpha_{s}^{n} \ln ^{n+1} N, g_{D Y}^{(2)}$ contains the next-to-leading logarithmic (NLL) terms $\alpha_{s}^{n} \ln ^{n} N, \alpha_{s} g_{D Y}^{(3)}$ contains the next-to-next-to-leading logarithmic (NNLL) terms $\alpha_{s}^{n+1} \ln ^{n} N$, and so forth. Once the functions $g_{D Y}^{(k)}$ have been computed, one has a systematic perturbative treatment of the region of $N$ in which $\alpha_{s} \ln N \lesssim 1$, which is much larger than the domain $\alpha_{s} \ln ^{2} N \ll 1$ in which the fixed-order calculation in $\alpha_{s}$ is reliable.

The physical bases for the soft-gluon exponentiation in the DY process are dynamics and kinematics factorizations $[1,2]$. Dynamics factorization follows from gauge invariance and unitarity: in the soft limit multi-gluon amplitudes fulfil generalized factorization formulae given in terms of a single-gluon emission probability. Kinematics factorization follows from the fact that the multi-gluon phase space in the appropriate soft limit can exactly be factorized by working in $N$ space.

The general extension of these results to the resummation of NLL contributions in heavyquark production was first considered by Kidonakis and Sterman [8] and more recently examined in 
ref. [9]. There are kinematics and dynamics complications in going from the DY to the heavy-quark processes. Although in the heavy-quark case the kinematics is more involved it can nonetheless be recast in factorized form in $N$-moment space $[8,9]$. Dynamics complications are more essential. While in the DY case resummation is only associated with the $q \bar{q}$-annihilation subprocess, in the heavy-quark case one has to consider both the $q \bar{q}$ and $g g$ subprocesses. Moreover, unlike the DY vector boson, the heavy-quark pair carries colour charge. Thus the heavy-quark cross-section is sensitive to radiative corrections due to soft-gluon emission from the heavy quark and antiquark produced in the final state. In particular, this emission induces colour exchange in the hard subprocess, whose colour state can thus fluctuate. The presence of colour fluctuations implies that, after soft-gluon resummation, the partonic cross-sections have an exponentiated form that is more involved than the simple exponential in eq. (3).

The general structure of the resummed partonic cross-section for heavy-quark hadroproduction is as follows [8]

$$
\hat{\sigma}_{i j, N}^{(r e s)}=\sum_{\mathbf{I}, \mathbf{J}} M_{i j, \mathbf{I}, N}^{\dagger}\left[\boldsymbol{\Delta}_{i j, N}\right]_{\mathbf{I}, \mathbf{J}} M_{i j, \mathbf{J}, N}
$$

where all the various factors depend on the heavy-quark mass $m$ and the renormalized coupling $\alpha_{s}\left(\mu^{2}\right)$ evaluated at the scale $\mu$, although this dependence is not explicitly denoted. The sum in eq. (4) is over all possible colour states $\mathbf{I}, \mathbf{J}$ of the LO hard-scattering subprocess $i+j \rightarrow Q \bar{Q}$. The colour amplitude $M_{i j, \mathbf{I}, N}$ is computable as a power series expansion in $\alpha_{s}$, but it does not contain any logarithmically-enhanced corrections produced by soft-gluon radiation. These corrections are embodied by the factor $\boldsymbol{\Delta}_{i j, N}$ that is a matrix in the space of the colour states. Performing the allorder resummation, the factor $\boldsymbol{\Delta}_{i j, N}$ can be expressed in exponential form but, unlike $\Delta_{D Y, N}$, we are now dealing with an exponential matrix and exponentiation has to be understood in a formal sense. The analogues of the functions $g_{D Y}^{(k)}$ are colour matrices. They have to be evaluated and then diagonalized, before one can rewrite the right-hand side of eq. (4) as an actual exponential.

The resummation formula (4) applies to the general case of heavy-quark production at fixed invariant mass of the produced quark pair and at fixed scattering angle ${ }^{4}$. In this case, the radiative factor $\boldsymbol{\Delta}_{i j, N}$ depends on the difference in rapidity between the heavy quark and antiquark and the NLL resummation has been worked out explicitly in ref. [8].

The goal of this paper is the resummation of logarithmically-enhanced corrections to the heavyquark total hadroproduction cross-section, up to NLL order. The non-trivial angular dependence of the eigenvalues and eigenvectors of the radiative factor $\boldsymbol{\Delta}_{i j, N}$ calculated in ref. [8], however, makes it difficult to apply in practice [11] this approach to the evaluation of the total production cross-section. A new general method for the resummation of soft-gluon contributions to hardscattering processes has recently been developed in refs. [9, 12]. This technique allows a more direct evaluation of the logarithmically-enhanced corrections to the heavy-quark total hadroproduction cross-section, up to NLL accuracy ${ }^{5}$. A key simplification in this method is the direct diagonalization of the radiative factor $\boldsymbol{\Delta}_{i j, N}$ for the resummed parton-level total cross-section in the colour-basis defined by colour-singlet and colour-octet final states. Details on the calculation and the illustration of the general method are presented elsewhere $[9,12]$. In this paper we limit ourselves to presenting the final theoretical results on soft-gluon resummation to NLL order, and we mainly concentrate on the ensuing phenomenological predictions.

\footnotetext{
${ }^{4} \mathrm{~A}$ similar structure also holds for the production of dijets at fixed invariant mass [10].

${ }^{5}$ We have checked that our resummation formulae are consistent with the NLL results on the heavy-quark cross-section at fixed invariant mass obtained in ref. [8].
} 
The rest of this paper is organized as follows. In Section 2 we describe our notation, and collect the fixed-order leading and next-to-leading formulae that will be used in the following. In Section 3 we give the formulae for the NLL resummed cross-sections, and in Sect. 4 we present our phenomenological results.

\section{Notation and fixed-order calculations}

In this Section we recall the known theoretical results $[13,14]$ on the calculation of the heavyquark hadroproduction cross-section at LO and next-to-leading-order (NLO) in QCD perturbation theory. We shall follow closely the notation of ref. [13].

The total cross-section $\sigma$ is given by the following factorization formula

$$
\sigma\left(\rho_{h}, m^{2}\right)=\sum_{i, j} \int_{0}^{1} d x_{1} d x_{2} F_{i}\left(x_{1}, \mu^{2}\right) F_{j}\left(x_{2}, \mu^{2}\right) \hat{\sigma}_{i j}\left(\rho ; m^{2}, \alpha_{s}\left(\mu^{2}\right), \mu^{2}\right)
$$

where $m$ is the mass of the heavy quark, $S$ is the square of the centre-of-mass energy, $i, j$ denote parton indices $(i=q, \bar{q}, g)$ and the dimensionless variables $\rho$ and $\rho_{h}$ are

$$
\rho_{h}=\frac{4 m^{2}}{S}, \quad \rho=\frac{\rho_{h}}{x_{1} x_{2}} .
$$

The parton densities $F_{i}\left(x, \mu^{2}\right)$ and the partonic cross-sections $\hat{\sigma}_{i j}$ depend on the factorization scale $\mu$ (which we always set equal to the renormalization scale) and on the factorization scheme. We use the $\overline{\mathrm{MS}}$ factorization scheme.

We also introduce the dimensionless functions $f_{i j}$ by rescaling the partonic cross-sections as follows

$$
\hat{\sigma}_{i j}\left(\rho ; m^{2}, \alpha_{s}\left(\mu^{2}\right), \mu^{2}\right) \equiv \frac{\alpha_{s}^{2}\left(\mu^{2}\right)}{m^{2}} f_{i j}\left(\rho ; \alpha_{s}\left(\mu^{2}\right), \mu^{2} / m^{2}\right) .
$$

The functions $f_{i j}$ are perturbatively computable and given by the following expansion in $\alpha_{s}$

$$
f_{i j}\left(\rho ; \alpha_{s}\left(\mu^{2}\right), \mu^{2} / m^{2}\right)=f_{i j}^{(0)}(\rho)+4 \pi \alpha_{s}\left(\mu^{2}\right)\left[f_{i j}^{(1)}(\rho)+\bar{f}_{i j}^{(1)}(\rho) \ln \frac{\mu^{2}}{m^{2}}\right]+\sum_{n=2}^{\infty} \alpha_{s}^{n}\left(\mu^{2}\right) f_{i j}^{(n)}\left(\rho ; \mu^{2} / m^{2}\right) \text {. }
$$

The LO terms $f_{i j}^{(0)}$ in eq. (8) are explicitly given by

$$
\begin{aligned}
f_{q \bar{q}}^{(0)}(\rho) & =\frac{\pi}{6} \frac{T_{R} C_{F}}{N_{c}} \beta \rho(2+\rho), \\
f_{g g}^{(0)}(\rho) & =\frac{\pi}{12} \frac{T_{R}}{N_{c}^{2}-1} \beta \rho\left\{3 C_{F}\left[\left(4+4 \rho-2 \rho^{2}\right) \frac{1}{\beta} \ln \frac{1+\beta}{1-\beta}-4-4 \rho\right]\right. \\
& \left.+C_{A}\left[3 \rho^{2} \frac{1}{\beta} \ln \frac{1+\beta}{1-\beta}-4-5 \rho\right]\right\},
\end{aligned}
$$

where $\beta \equiv \sqrt{1-\rho}$ and $f_{i j}^{(0)}(\rho)=0$ for all the other parton channels.

The NLO terms $\bar{f}_{i j}^{(1)}(\rho)$ in eq. (8) are obtained by simply performing the convolution of $f_{i j}^{(0)}(\rho)$ with Altarelli-Parisi splitting functions. The remaining NLO contributions $f_{i j}^{(1)}(\rho)$, first evaluated in ref. [13], are not known in analytic form but only numerically [13, 14]. An analytic parametrization of the numerical results is available in ref. [13]. The functions $f_{q \bar{q}}^{(1)}(\rho)$ and $f_{g g}^{(1)}(\rho)$ are plotted in fig. 1 (solid lines). 


\subsection{Threshold behaviour}

We are mainly interested in the behaviour of QCD corrections near the threshold region, $\rho \rightarrow 1$. In this region, the LO functions $f_{i j}^{(0)}(\rho)$ vanish because of phase-space suppression:

$$
f_{q \bar{q}}^{(0)}(\rho) \stackrel{\sim}{\rightrightarrows} 1 \frac{T_{R} C_{F}}{2 N_{c}} \pi \beta \rightarrow 0, \quad f_{g g}^{(0)}(\rho) \stackrel{\sim}{\rightrightarrows} 1 \frac{T_{R}}{N_{c}^{2}-1}\left(C_{F}-\frac{C_{A}}{4}\right) \pi \beta \rightarrow 0
$$

while the NLO functions do not. The behaviour of the latter is analytically known and given by the following expressions $[13,14]$

$$
\begin{aligned}
f_{q \bar{q}}^{(1)}(\rho)+\bar{f}_{q \bar{q}}^{(1)}(\rho) \ln \frac{\mu^{2}}{m^{2}} & =\frac{1}{4 \pi^{2}} f_{q \bar{q}}^{(0)}(\rho)\left\{\left(C_{F}-\frac{1}{2} C_{A}\right) \frac{\pi^{2}}{2 \beta}+2 C_{F} \ln ^{2}\left(8 \beta^{2}\right)\right. \\
& \left.-\left(8 C_{F}+C_{A}\right) \ln \left(8 \beta^{2}\right)-2 C_{F} \ln \left(4 \beta^{2}\right) \ln \frac{\mu^{2}}{m^{2}}+\bar{C}_{2}\left(\frac{\mu^{2}}{m^{2}}\right)+\mathcal{O}(1-\rho)\right\} \\
f_{g g}^{(1)}(\rho)+\bar{f}_{g g}^{(1)}(\rho) \ln \frac{\mu^{2}}{m^{2}} & =\frac{1}{4 \pi^{2}} f_{g g}^{(0)}(\rho)\left\{\frac{N_{c}^{2}+2}{N_{c}\left(N_{c}^{2}-2\right)} \frac{\pi^{2}}{4 \beta}+2 C_{A} \ln ^{2}\left(8 \beta^{2}\right)\right. \\
& \left.-\frac{\left(9 N_{c}^{2}-20\right) C_{A}}{N_{c}^{2}-2} \ln \left(8 \beta^{2}\right)-2 C_{A} \ln \left(4 \beta^{2}\right) \ln \frac{\mu^{2}}{m^{2}}+\bar{C}_{3}\left(\frac{\mu^{2}}{m^{2}}\right)+\mathcal{O}(1-\rho)\right\}
\end{aligned}
$$

and $f_{i j}^{(1)}(\rho), \bar{f}_{i j}^{(1)}(\rho)=\mathcal{O}(\beta)$ for all the other parton channels.

The first term in the curly brackets of eqs. $(12,13)$ is due to the Coulomb interaction between the heavy quark and antiquark. The remaining logarithmic contributions are produced by softgluon bremsstrahlung. Note that the right-hand sides of eqs. $(12,13)$ also include the constant coefficients $\bar{C}_{2}, \bar{C}_{3}$, which are due to large-momentum virtual corrections. The explicit expressions for these coefficients are

$$
\bar{C}_{2}\left(\frac{\mu^{2}}{m^{2}}\right)=C_{2}+\left(n_{l f}-4\right)\left(\frac{2}{3} \ln 2-\frac{5}{9}\right)+\left(\frac{53}{6}-\frac{1}{3} n_{l f}\right) \ln \frac{\mu^{2}}{m^{2}}, \quad \bar{C}_{3}\left(\frac{\mu^{2}}{m^{2}}\right)=C_{3}+12 \ln \frac{\mu^{2}}{m^{2}},
$$

where $n_{l f}$ denotes the number of massless flavours (i.e. the number of quark flavours that are lighter than the produced heavy-quark pair) and $C_{2,3}$ are obtained from the values of the constants $a_{0}$ reported in Table 1 of ref. [13]:

$$
C_{2}=36 \pi a_{0}^{q \bar{q}} \simeq 20.45, \quad C_{3}=\frac{768}{7} \pi a_{0}^{g g} \simeq 37.23
$$

In the strict threshold limit the Coulomb contributions dominate the radiative corrections in eqs. $(12,13)$. However, in most of the hadroproduction processes of phenomenological interest, the heavy-quark pair is not produced exactly at threshold. In these cases, it turns out $[13,14]$ that the logarithmic terms due to soft-gluon emission give the bulk of the NLO corrections. Multiple-gluon radiation at higher perturbative orders leads to more enhanced logarithmic corrections and the coefficient functions $f_{i j}^{(n)}\left(\rho ; \mu^{2} / m^{2}\right)$ in eq. (8) behave as

$$
f_{i j}^{(n)}\left(\rho ; \mu^{2} / m^{2}\right) \sim f_{i j}^{(0)}(\rho) \ln ^{2 n} \beta^{2} .
$$

Resummation of these soft-gluon effects to all orders in perturbation theory can be important to improve the reliability of the QCD predictions. 


\section{$2.2 \quad N$-moment space}

The resummation program of soft-gluon contributions has been carried out $[1,2]$ in the Mellintransform space, or $N$-space. Working in $N$-space, one can disentangle the soft-gluon effects in the parton densities from those in the partonic cross-section and one can straightforwardly implement and factorize the kinematic constraints of energy and longitudinal-momentum conservation.

The Mellin transform of the heavy-quark hadronic cross-section $\sigma\left(\rho_{h}, m^{2}\right)$ is defined as follows

$$
\sigma_{N}\left(m^{2}\right) \equiv \int_{0}^{1} d \rho \rho^{N-1} \sigma\left(\rho, m^{2}\right)
$$

where $N$ is the moment index. In $N$-moment space eq. (5) takes a simple factorized expression

$$
\begin{aligned}
\sigma_{N}\left(m^{2}\right) & =\sum_{i, j} F_{i, N+1}\left(\mu^{2}\right) F_{j, N+1}\left(\mu^{2}\right) \hat{\sigma}_{i j, N}\left(m^{2}, \alpha_{s}\left(\mu^{2}\right), \mu^{2}\right) \\
& =\sum_{i, j} F_{i, N+1}\left(\mu^{2}\right) F_{j, N+1}\left(\mu^{2}\right) \frac{\alpha_{s}^{2}\left(\mu^{2}\right)}{m^{2}} f_{i j, N}\left(\alpha_{s}\left(\mu^{2}\right), \mu^{2} / m^{2}\right),
\end{aligned}
$$

where we have used eq. (8) and we have introduced the $N$-moments $F_{i, N}\left(\mu^{2}\right)$ of the parton densities,

$$
F_{i, N}\left(\mu^{2}\right) \equiv \int_{0}^{1} d x x^{N-1} F_{i}\left(x, \mu^{2}\right)
$$

and likewise for any other $x$-space function.

The $N$-moments of the LO functions in eqs. (9) and (10) are given by the following explicit expressions [7]

$$
\begin{aligned}
f_{q \bar{q}, N}^{(0)} & =\frac{\pi^{\frac{3}{2}}}{4} \frac{T_{R} C_{F}}{N_{c}} \frac{\Gamma(N+1)}{\Gamma(N+7 / 2)}(N+2) \\
f_{g g, N}^{(0)} & =\frac{\pi^{\frac{3}{2}}}{4} \frac{T_{R}}{N_{c}^{2}-1} \frac{\Gamma(N+1)}{\Gamma(N+5 / 2)} \frac{1}{N+3} \\
& \times\left[2 C_{F} \frac{N^{3}+9 N^{2}+20 N+14}{(N+1)(N+2)}-C_{A} \frac{N^{2}+8 N+11}{2 N+5}\right]
\end{aligned}
$$

To discuss the structure of the higher-order corrections considered in the next Section, it is useful to decompose the various cross-section contributions according to the colour state (singlet $\mathbf{1}$ and octet $\mathbf{8}$ ) of the heavy-quark pair produced in the final state. When applied to the LO functions $f_{i j}^{(0)}$, this decomposition gives

$$
f_{i j}^{(0)}(\rho)=f_{i j, \mathbf{1}}^{(0)}(\rho)+f_{i j, \mathbf{8}}^{(0)}(\rho)
$$

with [6]

$$
\begin{aligned}
& f_{g g, \mathbf{1}}^{(0)}(\rho)=\frac{T_{R}}{N_{c}\left(N_{c}^{2}-1\right)} \frac{\pi \beta \rho}{8}\left[\left(4+4 \rho-2 \rho^{2}\right) \frac{1}{\beta} \ln \frac{1+\beta}{1-\beta}-4-4 \rho\right] \\
& f_{g g, \mathbf{8}}^{(0)}(\rho)=f_{g g}^{(0)}(\rho)-f_{g g, \mathbf{1}}^{(0)}(\rho), \quad f_{q \bar{q}, \mathbf{1}}^{(0)}(\rho)=0, \quad f_{q \bar{q}, \mathbf{8}}^{(0)}(\rho)=f_{q \bar{q}}^{(0)}(\rho)
\end{aligned}
$$


The $N$-moments of $f_{g g, \mathbf{1}}^{(0)}(\rho)$ are

$$
f_{g g, \mathbf{1}, N}^{(0)}=\frac{\pi^{\frac{3}{2}}}{4} \frac{T_{R}}{N_{c}\left(N_{c}^{2}-1\right)} \frac{\Gamma(N+1)}{\Gamma(N+5 / 2)} \frac{N^{3}+9 N^{2}+20 N+14}{(N+1)(N+2)(N+3)},
$$

and those of $f_{g g, \mathbf{8}}^{(0)}(\rho)$ and $f_{q \bar{q}, \boldsymbol{8}}^{(0)}(\rho)$ are then obtained from eqs. $(21,22)$ by using eq. $(24)$

We also introduce the functions $f_{i j}^{(1), \operatorname{Coul}}(\rho)$ that describe the Coulomb contributions to the NLO corrections in eq. $(12,13)$. Using the singlet/octet colour decomposition, we have

$$
\begin{aligned}
f_{i j, \mathbf{1}}^{(1), \text { Coul }}(\rho) & =f_{i j, \mathbf{1}}^{(0)}(\rho) C_{F} \frac{1}{8 \beta}, \\
f_{i j, \mathbf{8}}^{(1), \text { Coul }}(\rho) & =f_{i j, \mathbf{8}}^{(0)}(\rho)\left(C_{F}-\frac{C_{A}}{2}\right) \frac{1}{8 \beta},
\end{aligned}
$$

whose Mellin transforms are given by

$$
\begin{aligned}
f_{q \bar{q}, \mathbf{8}, N}^{(1), \text { Coul }} & =-\frac{\pi}{96} \frac{T_{R} C_{F}}{N_{c}^{2}} \frac{3 N+5}{(N+1)(N+2)}, \\
f_{g g, \mathbf{8}, N}^{(1), \text { Coul }} & =-\frac{\pi}{192} \frac{T_{R}}{N_{c}\left(N_{c}^{2}-1\right)}\left[N_{c}\left(3 I_{N+2}-\frac{4}{N+1}-\frac{5}{N+2}\right)\right. \\
& \left.+3 \frac{N_{c}^{2}-2}{N_{c}}\left(2 I_{N}+2 I_{N+1}-I_{N+2}-\frac{2}{N+1}-\frac{2}{N+2}\right)\right] \\
f_{g g, \mathbf{1}, N}^{(1), \text { Coul }} & =\frac{\pi}{64} \frac{T_{R}}{N_{c}^{2}}\left[2 I_{N}+2 I_{N+1}-I_{N+2}-\frac{2}{N+1}-\frac{2}{N+2}\right] .
\end{aligned}
$$

The function $I_{N}$ is defined by:

$$
I_{N}=\int_{0}^{1} d \rho \rho^{N} \frac{1}{\sqrt{1-\rho}} \ln \frac{1+\sqrt{1-\rho}}{1-\sqrt{1-\rho}}
$$

We can rewrite $I_{N}$ as follows

$$
I_{N}=\int_{0}^{1} d \rho \rho^{N} \frac{1}{\sqrt{1-\rho}}\left[\ln \frac{1}{\rho}+2 \ln (1+\sqrt{1-\rho})\right] .
$$

The integral of the first term can be done exactly, while to integrate the second term we first expand the logarithm of $1+\sqrt{1-\rho}$ as:

$$
\ln (1+x)=\sum_{n=1}^{4} \alpha_{n} x^{n}
$$

with $\alpha_{n}=0.9991,-0.4828,0.2477,-0.0712$ for $n=1, \ldots, 4$, and then perform the integral term by term. The expansion in eq. (33) is accurate to $10^{-4}$ in the needed range $0<x<1$. The final approximate result for $I_{N}$ is:

$$
I_{N} \simeq \sqrt{\pi} \frac{\Gamma(N+1)}{\Gamma(N+3 / 2)}[\psi(N+3 / 2)-\psi(N+1)]+2 \sum_{n=1}^{4} \alpha_{n} \frac{\Gamma(N+1) \Gamma\left(\frac{n+1}{2}\right)}{\Gamma(N+n / 2+3 / 2)} .
$$

The threshold region $\rho \rightarrow 1$ corresponds to the limit $N \rightarrow \infty$ in $N$-moment space. In this limit the Mellin transform of the threshold expansions $(12,13)$ of the NLO corrections can be 
easily computed. Having introduced the Coulomb contributions $f_{i j}^{(1), \text { Coul }}$, we can write the result as follows

$$
\begin{aligned}
f_{q \bar{q}, N}^{(1)}+\bar{f}_{q \bar{q}, N}^{(1)} \ln \frac{\mu^{2}}{m^{2}} & =\frac{1}{4 \pi^{2}} f_{q \bar{q}, N}^{(0)}\left\{2 C_{F} \ln ^{2} N+\left(C_{A}+4 C_{F} \gamma_{E}+2 C_{F} \ln \frac{\mu^{2}}{4 m^{2}}\right) \ln N\right. \\
& \left.+C_{q \bar{q}}\left(\mu^{2} / m^{2}\right)+\mathcal{O}\left(\frac{1}{N}\right)\right\}+f_{q \bar{q}, \mathbf{8}, N}^{(1), \text { Coul }} \\
f_{g g, N}^{(1)}+\bar{f}_{g g, N}^{(1)} \ln \frac{\mu^{2}}{m^{2}} & =\frac{1}{4 \pi^{2}} f_{g g, N}^{(0)}\left\{2 C_{A} \ln ^{2} N+\left[\frac{N_{c}^{2}-4}{N_{c}^{2}-2} C_{A}+4 C_{A} \gamma_{E}+2 C_{A} \ln \frac{\mu^{2}}{4 m^{2}}\right] \ln N\right. \\
& \left.+C_{g g}\left(\mu^{2} / m^{2}\right)+\mathcal{O}\left(\frac{1}{N}\right)\right\}+f_{g g, \mathbf{1}, N}^{(1), \text { Coul }}+f_{g g, \mathbf{8}, N}^{(1), \text { Coul }}
\end{aligned}
$$

where $\gamma_{E}=0.5772 \ldots$ is the Euler number and the constant coefficients $C_{q \bar{q}}, C_{g g}$ are related to those in eqs. $(14,15)$ :

$$
\begin{aligned}
C_{q \bar{q}}\left(\frac{\mu^{2}}{m^{2}}\right) & =\bar{C}_{2}\left(\frac{\mu^{2}}{m^{2}}\right)+2 C_{F}\left[\ln ^{2} 2+\left(\gamma_{E}-2\right) \ln \frac{\mu^{2}}{4 m^{2}}+\frac{\pi^{2}}{2}+\gamma_{E}\left(\gamma_{E}-4\right)\right] \\
& +\left(8 C_{F}+C_{A}\right)\left(\gamma_{E}-2-\ln 2\right), \\
C_{g g}\left(\frac{\mu^{2}}{m^{2}}\right) & =\bar{C}_{3}\left(\frac{\mu^{2}}{m^{2}}\right)+2 C_{A}\left[\ln ^{2} 2+\left(\gamma_{E}-2\right) \ln \frac{\mu^{2}}{4 m^{2}}+\frac{\pi^{2}}{2}+\gamma_{E}\left(\gamma_{E}-4\right)\right] \\
& +\frac{C_{A}\left(9 N_{c}^{2}-20\right)}{N_{c}^{2}-2}\left(\gamma_{E}-2-\ln 2\right) .
\end{aligned}
$$

The double and single logarithmic terms $\ln ^{2} N$ and $\ln N$ in eqs. $(35,36)$ are produced by softgluon radiation. These terms dominate the corrections in the curly brackets of eqs. $(35,36)$. The resummation of soft-gluon contributions to all orders in perturbation theory is considered in the following Section.

\section{Soft-gluon resummation}

The partonic cross-sections $\hat{\sigma}_{i j, N}$ with $i j \neq q \bar{q}, g g$ vanish at LO and are hence suppressed by a factor of $\alpha_{s}$ with respect to $\hat{\sigma}_{q \bar{q}, N}$ and $\hat{\sigma}_{g g, N}$. In the threshold or large- $N$ limit, this relative suppression is further enhanced by a factor of $\mathcal{O}(1 / N)$. Therefore, we make no attempt to resum soft-gluon corrections to these partonic channels and we restrict ourselves to $\hat{\sigma}_{q \bar{q}, N}$ and $\hat{\sigma}_{g g, N}$.

\subsection{Total heavy-quark cross-section to NLL accuracy: the radiative factors}

As discussed in the Introduction, the resummed radiative factor $\left[\boldsymbol{\Delta}_{i j, N}\right]_{\mathbf{I}, \mathbf{J}}$ in eq. (4) depends in general on the partonic subprocess (labelled by $i j$ ) and on its colour states (labelled by $\mathbf{I}, \mathbf{J}$ ). However, in the case of the threshold behaviour of the total cross section, most of the complications related to the colour structure can be easily overcome. Up to NLL accuracy, it turns out [9, 12] that the colour matrix $\boldsymbol{\Delta}_{i j, N}$ is diagonal with respect to the basis $\mathbf{I}=\{\mathbf{1}, \mathbf{8}\}, \mathbf{1}$ and $\mathbf{8}$ being the singlet and octet state of the produced heavy-quark pair. We can thus present the exponentiated formulae for its eigenvalues $\Delta_{i j, \mathbf{I}, N}$ in the two colour channels. 
We use a notation similar to that in ref. [2] to facilitate the comparison with the known results for the DY process. In the $\overline{\mathrm{MS}}$ factorization and renormalization schemes, the NLL resummed expressions for the radiative factors $\Delta_{i j, \mathbf{I}, N}$ are:

$$
\begin{aligned}
\ln \Delta_{q \bar{q}, \mathbf{1}, N} & =\int_{0}^{1} d z \frac{z^{N-1}-1}{1-z} \int_{\mu^{2}}^{4 m^{2}(1-z)^{2}} \frac{d q_{\perp}^{2}}{q_{\perp}^{2}} 2 A_{q}\left(\alpha_{s}\left(q_{\perp}^{2}\right)\right)+\mathcal{O}\left(\alpha_{s}\left(\alpha_{s} \ln N\right)^{k}\right) \\
\ln \Delta_{q \bar{q}, \mathbf{8}, N} & =\int_{0}^{1} d z \frac{z^{N-1}-1}{1-z}\left\{\int_{\mu^{2}}^{4 m^{2}(1-z)^{2}} \frac{d q_{\perp}^{2}}{q_{\perp}^{2}} 2 A_{q}\left(\alpha_{s}\left(q_{\perp}^{2}\right)\right)+D_{Q \bar{Q}}\left(\alpha_{s}\left(4 m^{2}(1-z)^{2}\right)\right)\right\} \\
& +\mathcal{O}\left(\alpha_{s}\left(\alpha_{s} \ln N\right)^{k}\right) \\
\ln \Delta_{g g, \mathbf{1}, N} & =\int_{0}^{1} d z \frac{z^{N-1}-1}{1-z} \int_{\mu^{2}}^{4 m^{2}(1-z)^{2}} \frac{d q_{\perp}^{2}}{q_{\perp}^{2}} 2 A_{g}\left(\alpha_{s}\left(q_{\perp}^{2}\right)\right)+\mathcal{O}\left(\alpha_{s}\left(\alpha_{s} \ln N\right)^{k}\right) \\
\ln \Delta_{g g, \mathbf{8}, N} & =\int_{0}^{1} d z \frac{z^{N-1}-1}{1-z}\left\{\int_{\mu^{2}}^{4 m^{2}(1-z)^{2}} \frac{d q_{\perp}^{2}}{q_{\perp}^{2}} 2 A_{g}\left(\alpha_{s}\left(q_{\perp}^{2}\right)\right)+D_{Q \bar{Q}}\left(\alpha_{s}\left(4 m^{2}(1-z)^{2}\right)\right)\right\} \\
& +\mathcal{O}\left(\alpha_{s}\left(\alpha_{s} \ln N\right)^{k}\right)
\end{aligned}
$$

where $A_{q}[15], A_{g}[16], D_{Q \bar{Q}}[9]$ are the following perturbative functions

$$
\begin{aligned}
A_{q}\left(\alpha_{s}\right) & =C_{F} \frac{\alpha_{s}}{\pi}\left[1+\frac{K}{2} \frac{\alpha_{s}}{\pi}\right]+\mathcal{O}\left(\alpha_{s}^{3}\right), \\
A_{g}\left(\alpha_{s}\right) & =C_{A} \frac{\alpha_{s}}{\pi}\left[1+\frac{K}{2} \frac{\alpha_{s}}{\pi}\right]+\mathcal{O}\left(\alpha_{s}^{3}\right), \\
D_{Q \bar{Q}}\left(\alpha_{s}\right) & =-C_{A} \frac{\alpha_{s}}{\pi}+\mathcal{O}\left(\alpha_{s}^{2}\right) .
\end{aligned}
$$

and the coefficient $K$ is given by:

$$
K=\left(\frac{67}{18}-\frac{\pi^{2}}{6}\right) C_{A}-\frac{10}{9} T_{R} N_{f}
$$

Some comments are in order.

The LL terms $\alpha_{s}^{n} \ln ^{n+1} N$ in eqs. (39-42) are obtained by neglecting the contribution of the function $D_{Q \bar{Q}}$ and by truncating $A_{q}\left(\alpha_{s}\right)$ and $A_{g}\left(\alpha_{s}\right)$ to their first order. One thus recovers the LL resummed results first derived in ref. [3].

The contributions of the functions $A_{q}, A_{g}, D_{Q \bar{Q}}$ have a direct physical interpretation. The function $A_{q}\left(A_{g}\right)$ measures soft and collinear radiation from the $q \bar{q}(g g)$ partonic channel in the initial state. This is the only contribution that appears in hadroproduction processes of colourless heavy-mass systems, such as the DY process $[1,2,17]$ or Higgs boson production via gluon fusion $[16,18]$.

The function $D_{Q \bar{Q}}$ is due to soft emission from the final-state heavy-quark pair. Since the final-state quarks are massive, they do not lead to collinear logarithms and the function $D_{Q \bar{Q}}$ only enters at NLL accuracy. At threshold, the heavy-quark pair acts as a single final-state system with invariant-mass squared $Q^{2}=4 m^{2}$ and with colour charge given by the total $Q \bar{Q}$ charge. Thus, its contribution to the radiative factors $\Delta_{i j, \mathbf{I}, N}$ vanishes in the colour-singlet channels (see eqs. $(39,41))$ and is proportional to $C_{A}$ and independent of the initial state in the colour-octet channels (see eqs. $(40,42)$ ).

Since we know the resummed radiative factors $\Delta_{i j, \mathbf{I}, N}$ only to NLL accuracy, we use the expressions (39-42) by explicitly carrying out the $z$ and $q_{\perp}^{2}$ integrals and neglecting terms beyond 
NLL. Thus, we write:

$$
\begin{aligned}
\Delta_{i j, \mathbf{I}, N}\left(\alpha_{s}\left(\mu^{2}\right), \mu^{2} / m^{2}\right) & =\exp \left\{\ln N g_{i j}^{(1)}\left(b_{0} \alpha_{s}\left(\mu^{2}\right) \ln N\right)+g_{i j, \mathbf{I}}^{(2)}\left(b_{0} \alpha_{s}\left(\mu^{2}\right) \ln N, \mu^{2} / m^{2}\right)\right. \\
& \left.+\mathcal{O}\left(\alpha_{s}^{2}\left(\alpha_{s} \ln N\right)^{k}\right)\right\} .
\end{aligned}
$$

The functions $g^{(1)}$ and $g^{(2)}$ resum the LL and NLL terms, respectively. They can be explicitly computed as in refs. $[2,7]$. We find:

$$
g_{q \bar{q}}^{(1)}(\lambda)=\frac{C_{F}}{\pi b_{0} \lambda}[2 \lambda+(1-2 \lambda) \ln (1-2 \lambda)], \quad g_{g g}^{(1)}(\lambda)=\frac{C_{A}}{C_{F}} g_{q \bar{q}}^{(1)}(\lambda)
$$

and

$$
\begin{aligned}
g_{q \bar{q}, \mathbf{1}}^{(2)}\left(\lambda, \mu^{2} / m^{2}\right) & =-\gamma_{E} \frac{2 C_{F}}{\pi b_{0}} \ln (1-2 \lambda)+\frac{C_{F} b_{1}}{\pi b_{0}^{3}}\left[2 \lambda+\ln (1-2 \lambda)+\frac{1}{2} \ln ^{2}(1-2 \lambda)\right] \\
& -\frac{C_{F} K}{2 \pi^{2} b_{0}^{2}}[2 \lambda+\ln (1-2 \lambda)]-\frac{C_{F}}{\pi b_{0}} \ln (1-2 \lambda) \ln \frac{\mu^{2}}{4 m^{2}}, \\
g_{g g, \mathbf{1}}^{(2)}\left(\lambda, \mu^{2} / m^{2}\right) & =\frac{C_{A}}{C_{F}} g_{q \bar{q}, \mathbf{1}}^{(2)}\left(\lambda, \mu^{2} / m^{2}\right), \\
g_{i j, \mathbf{8}}^{(2)}\left(\lambda, \mu^{2} / m^{2}\right) & =g_{i j, \mathbf{1}}^{(2)}\left(\lambda, \mu^{2} / m^{2}\right)-\frac{C_{A}}{2 \pi b_{0}} \ln (1-2 \lambda) .
\end{aligned}
$$

where $b_{0}, b_{1}$ are the first two coefficients of the QCD $\beta$-function

$$
b_{0}=\frac{11 C_{A}-4 T_{R} N_{f}}{12 \pi}, \quad b_{1}=\frac{17 C_{A}^{2}-10 C_{A} T_{R} N_{f}-6 C_{F} T_{R} N_{f}}{24 \pi^{2}} .
$$

Note that the LL function $g_{i j, N}^{(1)}$ depends on the initial-state partonic channel, but it is independent of the colour state.

It is common practice to study the scale dependence of the cross-section in order to assess the impact of neglected higher-order effects. Normally, in the fixed-order calculations, one considers the range $m / 2<\mu<2 m$. When the threshold region is approached, however, a new scale comes into play, namely the typical distance from threshold of the hard production process. Thus, the process is really a two scale process, and one is forced to use a wider scale range in order to estimate the error. When the NLO calculation is improved by LL resummation, this is no longer needed, since the large threshold logarithms have been properly resummed. Thus, one can still safely consider the range $m / 2<\mu<2 m$. Observe, however, that the inclusion of LL resummation does not bring a reduction of the scale dependence in the range $m / 2<\mu<2 m$. The improvement comes simply from the fact that in this case we are allowed to use scales of order $m$. On the other hand, when NLL resummation is used, one does expect a reduction of the scale dependence in the range $m / 2<\mu<2 m$. This can be seen explicitly from eqs. (47-49). A scale variation from $\mu$ to $\mu^{\prime}$ is perturbatively equivalent to the replacement $\alpha_{s}\left(\mu^{2}\right) \rightarrow \alpha_{s}\left(\mu^{\prime 2}\right)=\alpha_{s}\left(\mu^{2}\right)\left[1-b_{0} \alpha_{s}(\mu) \ln \mu^{\prime 2} / \mu^{2}+\ldots\right]$. This replacement in the LL functions $g_{i j}^{(1)}\left(b_{0} \alpha_{s} \ln N\right)$ leads to additional NLL terms in the exponent on the right-hand side of eq. (47). These additional terms are partly cancelled by those that originate from the explicit $\mu$-dependence of the functions $g_{i j \mathbf{I} \mathbf{I}}^{(2)}\left(b_{0} \alpha_{s} \ln N, \mu^{2} / m^{2}\right)$. The left-over NLL contributions exactly match the scale dependence of the parton densities at large values of $N$. For instance, in the $q \bar{q}$ channel we have

$$
\Delta_{q \bar{q}, \mathbf{I}, N}\left(\alpha_{s}\left(\mu^{\prime 2}\right), \frac{\mu^{\prime 2}}{m^{2}}\right)=\Delta_{q \bar{q}, \mathbf{I}, N}\left(\alpha_{s}\left(\mu^{2}\right), \frac{\mu^{2}}{m^{2}}\right) \exp \left\{2 \frac{C_{F}}{\pi} \alpha_{s}\left(\mu^{2}\right) \ln N \ln \frac{\mu^{\prime 2}}{\mu^{2}}+\mathcal{O}\left(\alpha_{s}\left(\alpha_{s} \ln N\right)^{k}\right)\right\},
$$


and, in the calculation of the full hadronic cross-section, the exponential on the right-hand side is compensated by the scale dependence of the quark densities in the $\overline{\mathrm{MS}}$ factorization scheme:

$$
F_{q, N}\left(\mu^{\prime 2}\right) F_{\bar{q}, N}\left(\mu^{\prime 2}\right)=F_{q, N}\left(\mu^{2}\right) F_{\bar{q}, N}\left(\mu^{2}\right) \exp \left\{-2 \frac{C_{F}}{\pi} \alpha_{s}\left(\mu^{2}\right) \ln N \ln \frac{\mu^{\prime 2}}{\mu^{2}}+\mathcal{O}\left(\alpha_{s}\left(\alpha_{s} \ln N\right)^{k}\right)\right\} .
$$

\subsection{NLL resummed cross-section}

Using the results of Sect. 3.1, we can introduce the NLL resummed partonic cross-sections as follows

$$
\begin{gathered}
\hat{\sigma}_{i j, N}^{(r e s)}\left(m^{2}, \alpha_{s}\left(\mu^{2}\right), \mu^{2}\right)=\frac{\alpha_{s}^{2}\left(\mu^{2}\right)}{m^{2}} \sum_{\mathbf{I}=\mathbf{1}, \mathbf{8}} f_{i j, \mathbf{I}, N}^{(r e s)}\left(\alpha_{s}\left(\mu^{2}\right), \mu^{2} / m^{2}\right) \\
f_{i j, \mathbf{I}, N}^{(r e s)}\left(\alpha_{s}\left(\mu^{2}\right), \mu^{2} / m^{2}\right)=f_{i j, \mathbf{I}, N}^{(c o r r)}\left(\alpha_{s}\left(\mu^{2}\right), \mu^{2} / m^{2}\right) \Delta_{i j, \mathbf{I}, N+1}\left(\alpha_{s}\left(\mu^{2}\right), \frac{\mu^{2}}{m^{2}}\right) .
\end{gathered}
$$

Note the mismatch between the moment indices in the right-hand side of eq. (54). The radiative factors $\Delta_{i j, \mathbf{I}, N+1}$ depend on the moment index $N+1$, like the parton densities in the factorization formula (19).

The terms $f_{i j, \mathbf{I}, N}^{(c o r r)}$ in eq. (54) are given by the the LO functions $f_{i j, \mathbf{I}}^{(0)}$ in eqs. (23-25) after correction by the Coulomb contributions in eqs. $(28,29,30)$ and by the $N$-independent coefficients $C_{i j}$ in eqs. $(37,38)$

$$
f_{i j, \mathbf{I}, N}^{(c o r r)}=\left(f_{i j, \mathbf{I}, N}^{(0)}+4 \pi \alpha_{s}\left(\mu^{2}\right) f_{i j, \mathbf{I}, N}^{(1), \text { Coul }}\right)\left[1+\frac{\alpha_{s}\left(\mu^{2}\right)}{\pi} C_{i j}\left(\mu^{2} / m^{2}\right)\right] .
$$

Using the definition in eq. (55) and the perturbative expansions of the radiative factors $\Delta_{i j, \mathbf{I}, N}$,

$$
\begin{aligned}
\ln \Delta_{q \bar{q}, \mathbf{1}, N} & =\frac{C_{F}}{C_{A}} \ln \Delta_{g g, \mathbf{1}, N}=\frac{\alpha_{s}\left(\mu^{2}\right)}{\pi}\left[2 C_{F} \ln ^{2} N+\left(4 C_{F} \gamma_{E}+2 C_{F} \ln \mu^{2} / m^{2}\right) \ln N\right]+\mathcal{O}\left(\alpha_{s}^{2}\right), \\
\ln \Delta_{i j, \mathbf{8}, N} & =\ln \Delta_{i j, \mathbf{1}, N}+\frac{\alpha_{s}\left(\mu^{2}\right)}{\pi} C_{A} \ln N+\mathcal{O}\left(\alpha_{s}^{2}\right)
\end{aligned}
$$

it is straightforward to check that eqs. $(53,54)$ correctly reproduce the NLO threshold behaviour in eqs. $(35,36)$. In particular, since both the singlet and octet states are produced at LO via $g g$ fusion, the colour factor $\left(N_{c}^{2}-4\right) /\left(N_{c}^{2}-2\right)$ that appears in eq. (36) is due to the fact that final-state soft-gluon radiation is weighted by the ratio

$$
\frac{f_{g g, \mathbf{8}, N}^{(0)}}{f_{g g, N}^{(0)}}=\frac{N_{c}^{2}-4}{N_{c}^{2}-2}(1+\mathcal{O}(1 / N))
$$

which differs from unity near threshold.

The all-order factorization of $f_{i j, \mathbf{I}, N}^{(\text {corr })}$ with respect to the radiative factors $\Delta_{i j, \mathbf{I}, N+1}$ in the resummed partonic cross-sections (54) is justified by the fact that the $\mathcal{O}\left(\alpha_{s}\right)$-terms in eq. (55) are produced by non-soft virtual corrections to the LO subprocesses. The resummation of Coulomb corrections was considered in ref. [6] and it turned out that, in the experimental configurations of practical interest, its quantitative effect is much smaller than that due to soft-gluon radiation. 
Because of this reason, in our resummed formulae we do not include pure Coulomb effects beyond NLO, but simply the soft-gluon corrections to the $O\left(\alpha_{s}^{3}\right)$ Coulomb contribution.

Note, however, that we include the constant terms $C_{i j}\left(\mu^{2} / m^{2}\right)$ as in eqs. $(54,55)$. This procedure is analogous to that used in NLL resummed predictions for other observables, such as $e^{+} e^{-}$ event shapes [19]. As shown in Sect. 4.1, these constant terms are important to accurately match the exact near-threshold behavior at NLO. Moreover, when combined with the NLL soft-gluon factors $\Delta_{i j, \mathbf{I}, N}$, they lead to the following expansion

$$
\begin{aligned}
{\left[1+\frac{\alpha_{s}\left(\mu^{2}\right)}{\pi} C_{i j}\left(\mu^{2} / m^{2}\right)\right] \Delta_{i j, \mathbf{I}, N}\left(\alpha_{s}\left(\mu^{2}\right), \mu^{2} / m^{2}\right) } & =1+\sum_{n=1}^{+\infty} \alpha_{s}^{n}\left(\mu^{2}\right)\left[c_{n, 2 n} \ln ^{2 n} N+c_{n, 2 n-1} \ln ^{2 n-1} N\right. \\
& \left.+c_{n, 2 n-2}\left(\mu^{2} / m^{2}\right) \ln ^{2 n-2} N+\mathcal{O}\left(\ln ^{2 n-3} N\right)\right],(58)
\end{aligned}
$$

that correctly predicts the value of the coefficients $c_{n, 2 n-2}\left(\mu^{2} / m^{2}\right)$, as well as of $c_{n, 2 n}$ and $c_{n, 2 n-1}$ that are independent of $C_{i j}$. Note also that, while $c_{n, 2 n}$ and $c_{n, 2 n-1}$ are $\mu$-independent, the coefficients $c_{n, 2 n-2}\left(\mu^{2} / m^{2}\right)$ are not. Their dependence on $\mu$ is obtained by combining that of $C_{i j}\left(\mu^{2} / m^{2}\right)$ with the explicit scale dependence of $\Delta_{i j, \mathbf{I}, N}\left(\alpha_{s}\left(\mu^{2}\right), \mu^{2} / m^{2}\right)$ at NLL order.

Including the $C_{i j}$ constant term can also be viewed as using the known NLO cross-section to improve the resummation formula beyond the NLL approximation. This can be seen schematically in the following way. We write the left-hand side of eq. (58) as:

$$
\ln \left[\left(1+\alpha_{s} / \pi C\right) \Delta\right]=\ln \Delta+\alpha_{s} / \pi C,
$$

and since our NLL expression for $\ln \Delta$ has the form

$$
\ln \Delta=\ln N g^{(1)}\left(\alpha_{s} \ln N\right)+g^{(2)}\left(\alpha_{s} \ln N\right),
$$

according to eq. (3), the term $\alpha_{s} / \pi C$ corresponds precisely to the $O\left(\alpha_{s}\right)$ term in the expansion of the NNLL contribution $\alpha_{s} g^{(3)}\left(\alpha_{s} \ln N\right)$.

We use soft-gluon resummation to NLL accuracy to introduce an improved hadronic crosssection $\sigma_{N}^{(r e s)}$ as follows

$$
\begin{aligned}
\sigma_{N}^{(r e s)}\left(m^{2}\right) & =\sum_{i j=q \bar{q}, g g} F_{i, N+1}\left(\mu^{2}\right) F_{j, N+1}\left(\mu^{2}\right)\left[\hat{\sigma}_{i j, N}^{(r e s)}\left(m^{2}, \alpha_{s}\left(\mu^{2}\right), \mu^{2}\right)-\left(\hat{\sigma}_{i j, N}^{(r e s)}\left(m^{2}, \alpha_{s}\left(\mu^{2}\right), \mu^{2}\right)\right)_{\alpha_{s}^{3}}\right] \\
& +\sigma_{N}^{(N L O)}\left(m^{2}\right)
\end{aligned}
$$

where $\sigma_{N}^{(N L O)}$ is the hadronic cross-section at NLO, $\hat{\sigma}_{i j, N}^{(r e s)}$ is given in eq. (53) and $\left(\hat{\sigma}_{i j, N}^{(r e s)}\right)_{\alpha_{s}^{3}}$ represents its perturbative truncation at order $\alpha_{s}^{3}$. Thus, because of the subtraction in the square bracket on the right-hand side, eq. (61) exactly reproduces the NLO results and resums soft-gluon effects beyond $\mathcal{O}\left(\alpha_{s}^{4}\right)$ to NLL accuracy. This defines our NLO+NLL predictions.

The resummed formulae presented so far are given in $N$-moment space. To obtain cross-sections in the physical $x$-space, one has to perform the inverse Mellin transformation:

$$
\sigma^{(\mathrm{res})}\left(\rho, m^{2}\right)=\frac{1}{2 \pi i} \int_{C_{\mathrm{MP}}-i \infty}^{C_{\mathrm{MP}}+i \infty} d N \rho^{-N} \sigma_{N}^{(\mathrm{res})}\left(m^{2}\right) .
$$

When the $N$-moments $\sigma_{N}$ are evaluated at a fixed perturbative order in $\alpha_{s}$, they are analytic functions in a right half-plane of the complex variable $N$. In this case, the constant $C_{\mathrm{MP}}$ that 
defines the integration contour in eq. (62) has to be chosen in this half-plane, i.e. on the right of all the possible singularities of the $N$-moments.

An additional complication occurs when the $N$-moments are computed in resummed perturbation theory. In this case, since the resummed functions $g_{i j \mathbf{I}}^{(k)}(\lambda)$ in eqs. $(48,49)$ are singular at $\lambda=1 / 2$, the soft-gluon factors $\Delta_{i j, \mathbf{I}, N}\left(\alpha_{s}\left(\mu^{2}\right), \mu^{2} / m^{2}\right)$ in eq. (47) have cut singularities that start at the branch-point $N=N_{L}=\exp \left(1 / 2 b_{0} \alpha_{s}\right)$. These singularities, which are produced in eqs. (39-42) by the $q_{\perp}$-integration down to the Landau pole of the running coupling $\alpha_{s}\left(q_{\perp}^{2}\right)$, signal the onset of non-perturbative phenomena at very large values of $N$ or, equivalently, in the region very close to threshold.

The issue of how to deal with the Landau singularity in soft-gluon resummation formulae for hadronic collisions was discussed in detail in ref. [7]. In the evaluation of the inverse Mellin transformation (62) we thus use the Minimal Prescription introduced in ref. [7]. The constant $C_{\mathrm{MP}}$ is chosen in such a way that all singularities in the integrand are to the left of the integration contour, except for the Landau singularity at $N=N_{L}$, which should lie to the far right. This prescription is consistent [7] with the perturbative content of the soft-gluon resummation formulae because it converges asymptotically to the perturbative series and it does not introduce (unjustified) power corrections of non-perturbative origin. These corrections are certainly present in physical cross-sections, but their effect is not expected to be sizeable as long as $m$ is sufficiently perturbative and $\rho$ is sufficiently far from the hadronic threshold. Obviously, approaching the essentially non-perturbative regime $m \rightarrow 1 \mathrm{GeV}, \rho \rightarrow 1$, a physically motivated treatment of nonperturbative effects has to be introduced. In the following Section, we limit ourselves to present numerical and phenomenological results for kinematic configurations in which non-perturbative corrections should be smaller than the estimated uncertainty of the perturbative predictions.

In all cases considered, we have verified that the minimal prescription is in practice equivalent to evaluate eq. (62) order by order in perturbation theory, until the corrections become numerically insignificant. An example of this procedure will also be shown in Section 4.2.

\section{Results}

We present in this Section some numerical results, to provide an illustration of the size of the effects considered and a new estimate of heavy-quark production cross-sections of relevance for present and future hadronic colliders.

\subsection{Parton-level results}

We start by discussing the resummation effects at the level of partonic cross sections. The resummed partonic cross-section for the production of a heavy quark pair can be obtained from eqs. (61) and (62) by assuming structure functions of the form $F(x)=\delta(1-x)$, and therefore $F_{N}=1$ for all complex values of $N$.

We consider first the $O\left(\alpha_{s}^{3}\right)$ terms in the expansion of the resummed cross-section, in order to estimate to which accuracy this reproduces the exact NLO results. In fig. 1 (left) we plot the function $f_{q \bar{q}}^{(1)}$, defined in eq. (8), as a function of $\eta=(1-\rho) / \rho$. The exact $O\left(\alpha_{s}^{3}\right)$ result [13] is compared with three possible implementations of the resummation procedure, all equivalent at 


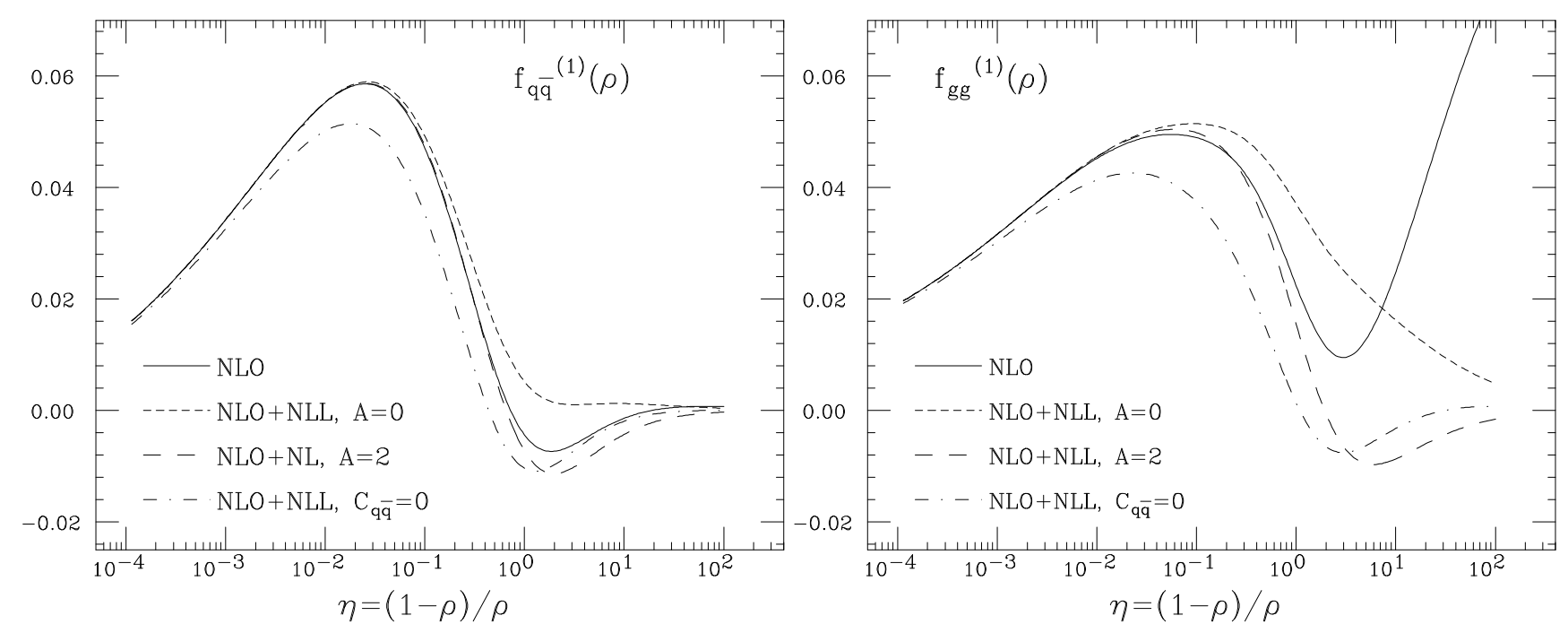

Figure 1: Left (Right): the function $f_{q \bar{q}}^{(1)}(\rho)\left(f_{g g}^{(1)}(\rho)\right)$, plotted as a function of $\eta=$ $(1-\rho) / \rho$. The solid line represents the exact NLO result $[13,14]$; the short-dashed line corresponds to the $O\left(\alpha_{s}^{3}\right)$ truncation of the resummed result defined by eqs. (53-55); the dot-dashed line is obtained from this last result by setting the constant $C_{q \bar{q}}\left(C_{g g}\right)$ to 0 ; the dashed line is obtained instead by the replacement in eq. (63), with $A=2$.

NLL. In one case (dot-dashed line) we set the constant $C_{q \bar{q}}$ introduced in eqs.(37) and (55) equal to 0 . In the second case (short-dashed line) we include the contribution of $C_{q \bar{q}}$. In a third case we correct the contribution of the constant $C_{q \bar{q}}$ by a term which is suppressed by a factor of $1 / N$, which does not introduce poles on the real $N$ axis, and which gives vanishing first moment to $f_{q \bar{q}, N}^{(1)}$ :

$$
C_{i j} \rightarrow C_{i j}\left(1-\frac{A}{N+A-1}\right), \quad i j=q \bar{q}, g g .
$$

In our applications we shall consider the two cases with $A=0$ (namely no correction to the $C_{i j}$ term) and $A=2$ as a way to establish the size of subleading corrections beyond the NLL order. In all cases we include the effect of the leading-order Coulomb effects, as described in eq. (55).

As one can see from fig. 1, the inclusion of the finite term $C_{q \bar{q}}$ is essential to accurately reproduce shape and normalization of the function $f_{q \bar{q}}^{(1)}$ near threshold. The agreement deteriorates unavoidably for $\rho \gg 1$, as here terms subleading in $1 / N$ become important. Note that the two choices $A=0$ and $A=2$ braket the exact result, and thus provide a good estimator of the subleading terms' systematics. The choice $A=2$, furthermore, provides a very accurate description up to values of $\eta$ of order 1 . This is the region which dominates the production cross-section in the cases of interest.

Analogous results for the $g g$ channel are given in the right panel of fig. 1 . The agreement is again very good near threshold. Far above threshold the exact NLO result is dominated by the $t$-channel gluon exchange diagrams $[13,20]$, which give rise to a $1 / N$ pole not controlled by the soft-gluon resummation.

The fully resummed parton-level cross-sections are shown in fig. 2 for the $q \bar{q}$ and $g g$ channels (left and right panel, respectively). Here and in the following we shall define the resummed cross-sections as in eq. (61), that is, we substitute their $O\left(\alpha_{s}^{3}\right)$ terms with the exact NLO result, using the same choice of renormalization scale $\mu$. In this way our results are exact up to (and 

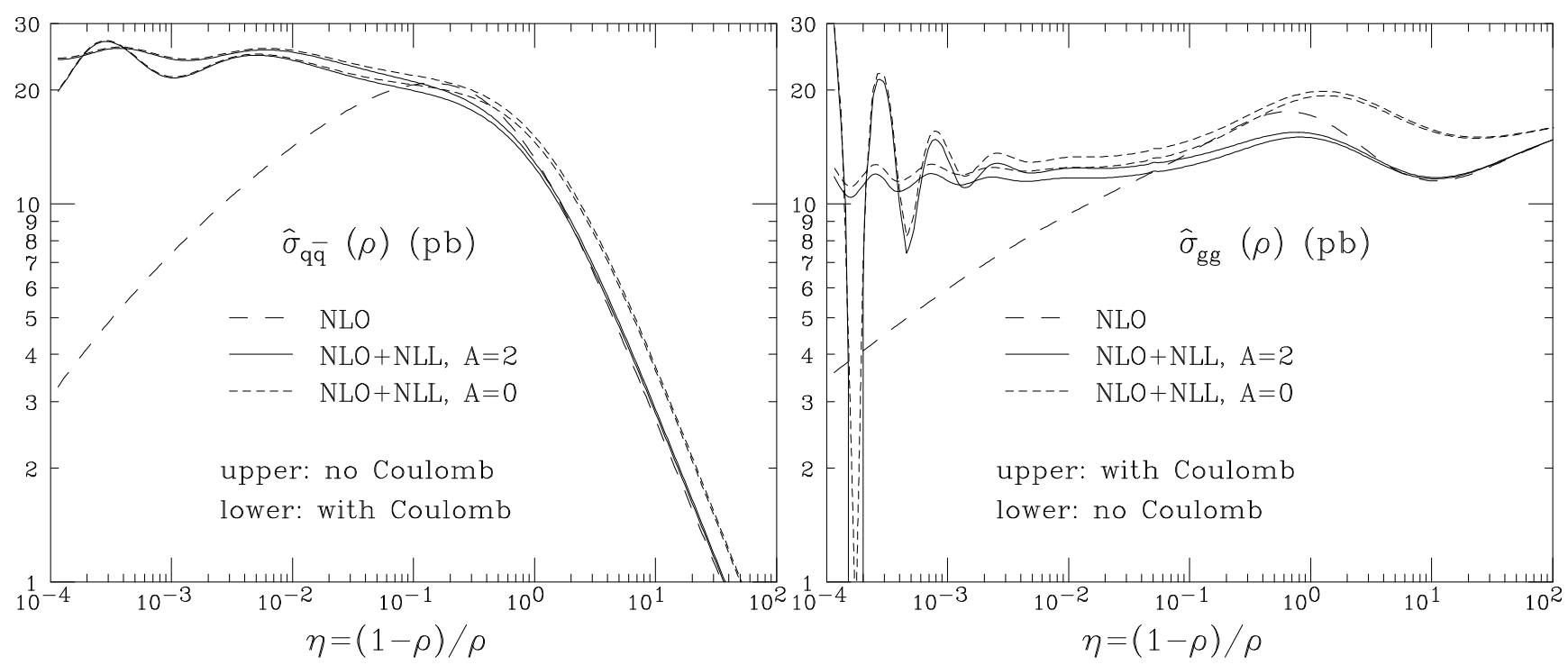

Figure 2: Partonic cross-section for the processes $q \bar{q} \rightarrow Q \bar{Q}$ (left) and $g g \rightarrow Q \bar{Q}$ (right) (in $\mathrm{pb}$, and for $m_{Q}=175 \mathrm{GeV}$ ). The dashed line is the exact NLO result $[13,14]$; the short-dashed (solid) lines correspond to the NLO+NLL result, with the coefficient $A$ defined in eq. (63) equal to 0 (2). The lower and upper curves correspond to inclusion or neglect of the Coulomb contribution.

including) $O\left(\alpha_{s}^{3}\right)$, and include the NLL resummation of terms of $O\left(\alpha_{s}^{4}\right)$ and higher. We compare the fixed-order results (dashed lines) with the resummed results. For these we provide both the $A=0$ and $A=2$ prescriptions, as well as the inclusion and neglect of the Coulomb terms in eq. (55). Note that even at the level of resummed cross-sections the prescriptions $A=0$ and $A=2$ braket the fixed order result. Note furthermore that, for the curves without the Coulomb contribution, the oscillations of the cross-section near threshold are significantly reduced relative to the LL resummation which was presented in fig. 3 of ref. [7].

\subsection{Hadron-level results}

In this Section we present results for the full hadronic cross-sections. As a default set of parton densities, we shall use the MRSR2 set described in [21]. For the top-quark mass we shall use, unless otherwise indicated, $m_{t}=175 \mathrm{GeV}$.

Figures 3, 4 and 5 present the ratios:

$$
\frac{\sigma_{\mathrm{NLL}, \mathrm{gg}}^{\mathrm{res}}-\sigma_{\mathrm{gg}}^{\mathrm{NLO}}}{\sigma_{\mathrm{gg}}^{\mathrm{NLO}}}, \quad \frac{\sigma_{\mathrm{NLL}, \mathrm{q} \overline{\mathrm{q}}}^{\mathrm{res}}-\sigma_{\mathrm{q} \overline{\mathrm{q}}}^{\mathrm{NLO}}}{\sigma_{\mathrm{q} \overline{\mathrm{q}}}^{\mathrm{NLO}}}, \frac{\sigma_{\mathrm{NLL},(\mathrm{gg}+\mathrm{q} \overline{\mathrm{q}})}^{\mathrm{res}}-\sigma_{(\mathrm{gg}+\mathrm{q} \overline{\mathrm{q}})}^{\mathrm{NLO}}}{\sigma_{(\mathrm{gg}+\mathrm{q} \overline{\mathrm{q}})}^{\mathrm{NLO}}} .
$$

For each channel we present the results using both the $A=0$ (dashed lines) and $A=2$ (solid lines) prescriptions. We also show the dependence on the choice of renormalization and factorization scales, which we always take equal, and varying within the set $\mu=\left(m_{t} / 2, m_{t}, 2 m_{t}\right)$. Note that the size of the resummation effects is larger for the larger scales, contrary to the behaviour of the scale dependence of the NLO cross section. This suggests that the scale dependence of the resummed cross-section will be reduced relative to that of the NLO results. The size of the resummation corrections is large at small centre-of-mass energies, as should be expected for the region where production is dominated by the threshold region. At Tevatron energies $(\sqrt{S}=1.8 \mathrm{TeV})$, the size 


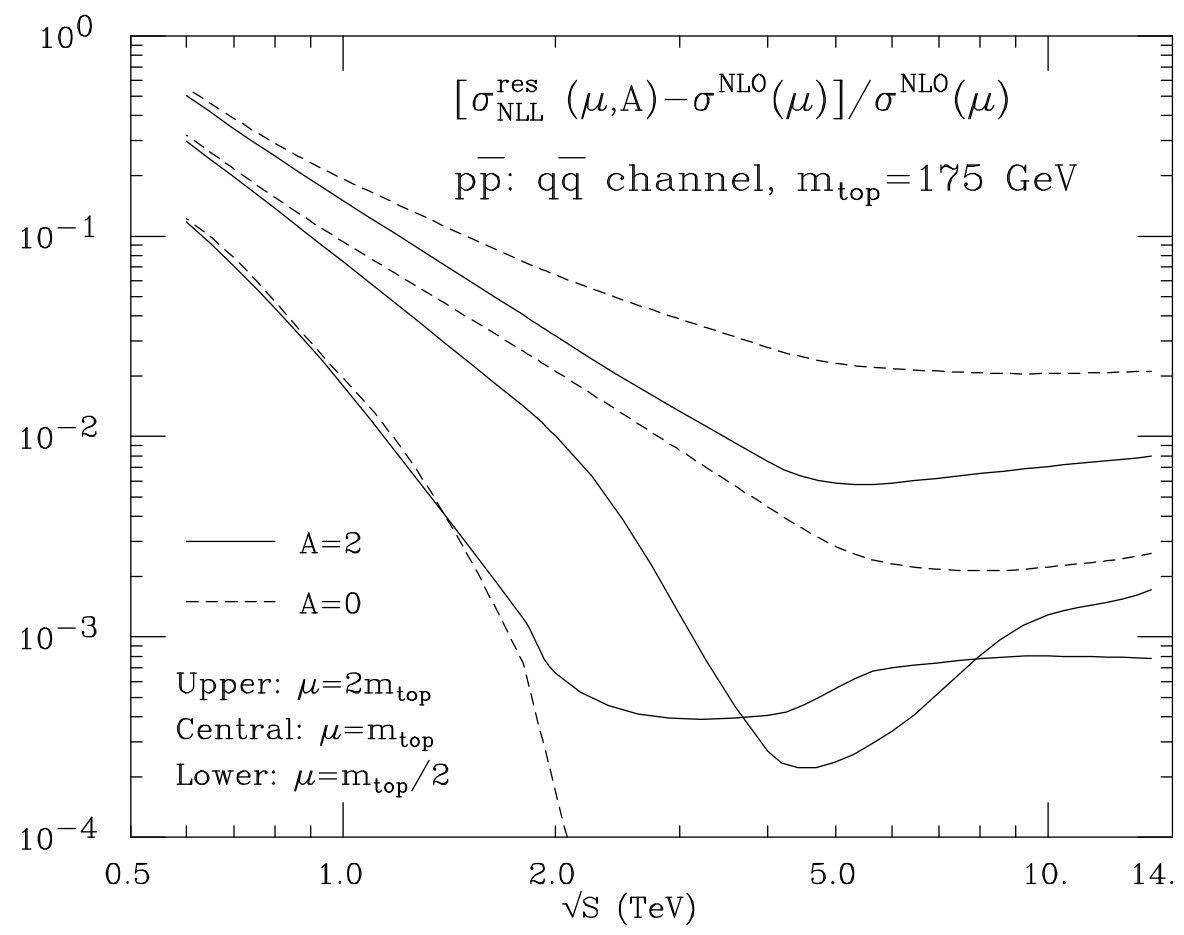

Figure 3: Contribution of gluon resummation at order $O\left(\alpha_{s}^{4}\right)$ and higher, relative to the exact NLO result, for top-pair production via $q \bar{q}$ annihilation in $p \bar{p}$ collisions. The solid (dashed) lines correspond to $A=2(A=0)$. The three sets of curves correspond to the choice of scale $\mu=2 m_{t}, m_{t}$ and $m_{t} / 2$, in descending order, with $m_{t}=175 \mathrm{GeV}$, and PDF set MRSR2.

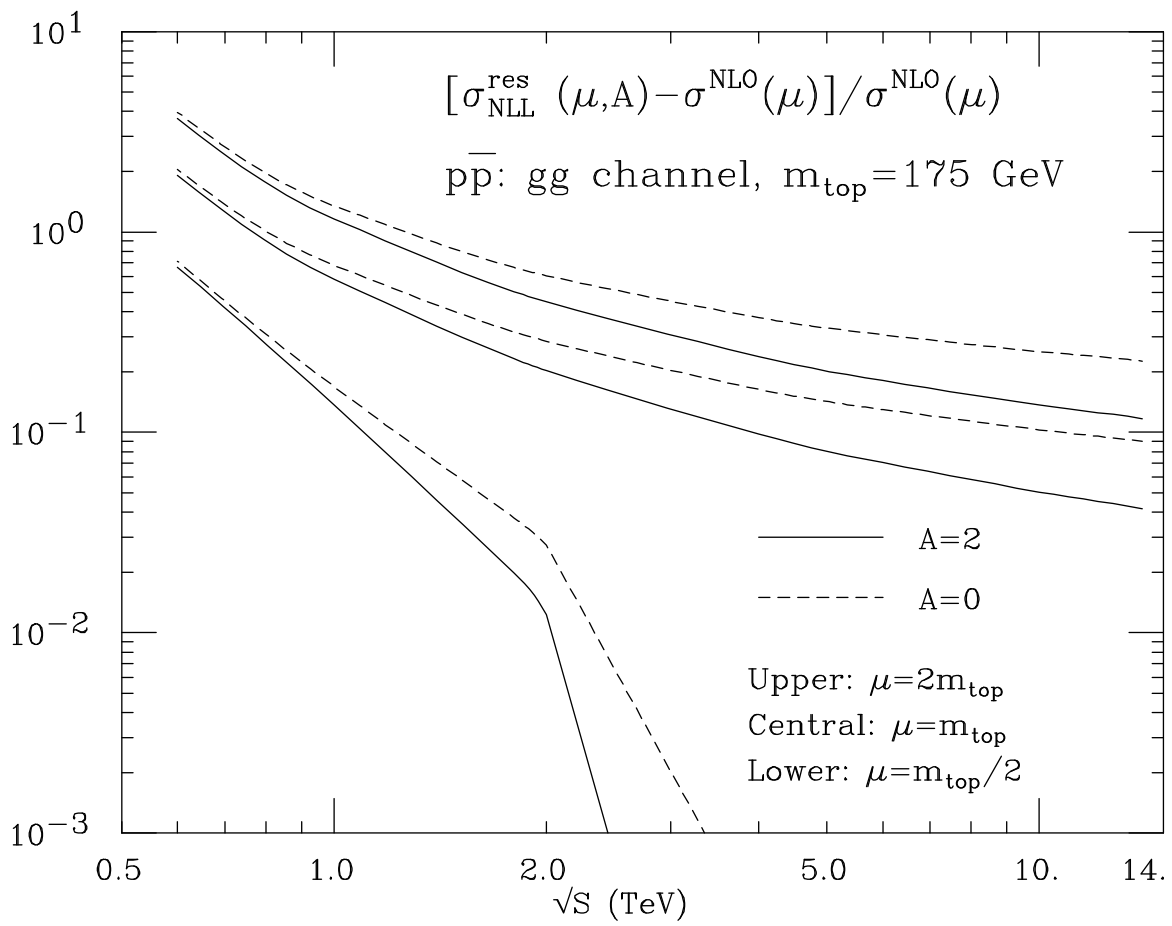

Figure 4: Same as fig. 3, for production via $g g$ annihilation. 


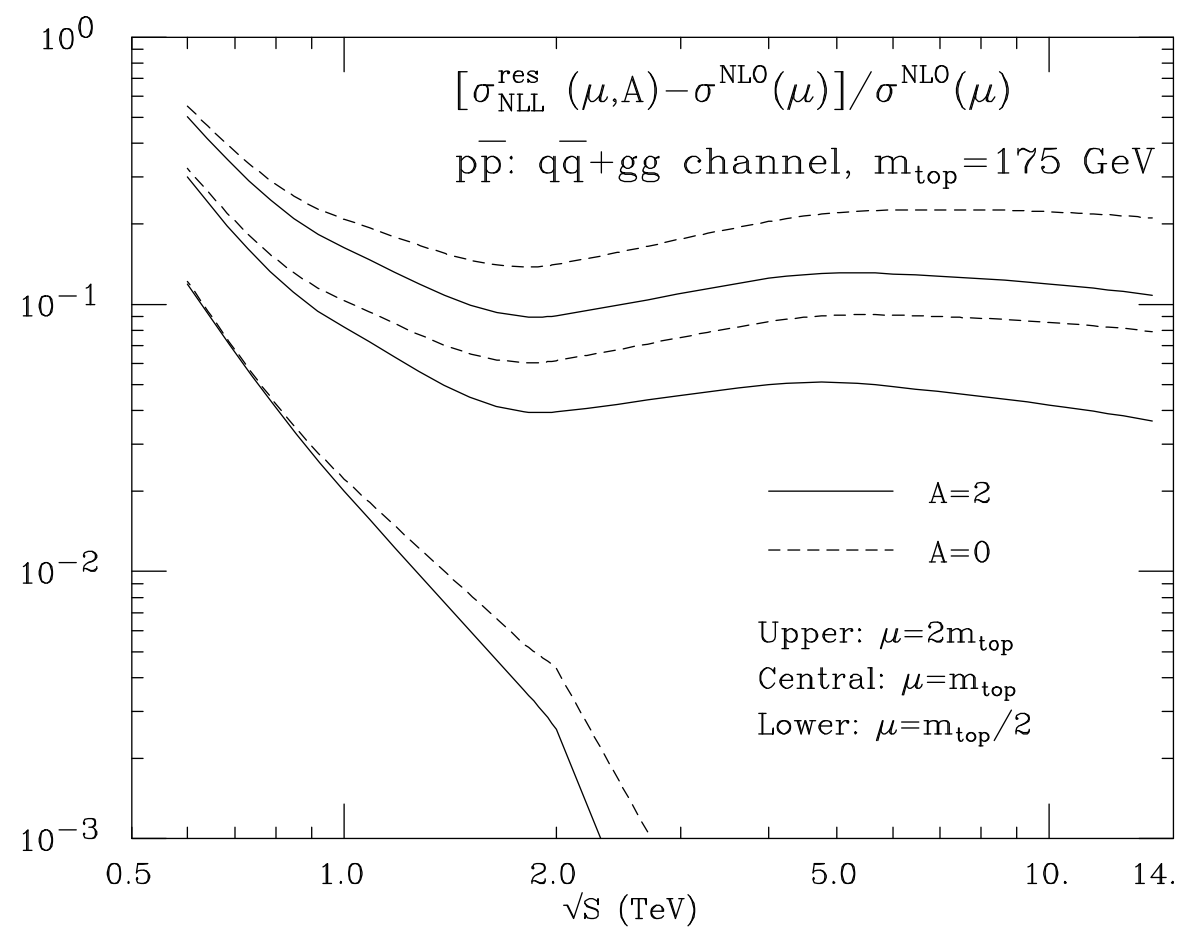

Figure 5: Same as fig. 3, for the combined production channels $g g+q \bar{q}$.

of the corrections equals $4 \%(6 \%)$ for $A=2(A=0)$ and $\mu=m_{t}$. It becomes equal to $0.4 \%$ $(0.5 \%)$ for $\mu=m_{t} / 2$ and to $9 \%$ (14\%) for $\mu=2 m_{t}$.

The scale dependence of the resummed cross-section, compared to the NLO one, is given in figs. 6 and 7 , corresponding to the $A=0$ and $A=2$ cases, respectively. Note the significant reduction in scale dependence, more marked in the $A=0$ case. More importantly, note that the band of variation of the resummed cross section lies entirely within the band of variation of the NLO cross-section for Tevatron energies and above. This shows that previous estimates of the theoretical systematic uncertainty for the Tevatron cross-section were correct, and can now be improved thanks to the NLL calculation presented here.

To display the importance of the inclusion of the NNLL $C_{i j}$ terms, we show the same plot of the scale dependence with $C_{i j}=0$ in fig. 8 . While the scale sensitivity is slightly worse than in the cases with $C_{i j} \neq 0$, there is still an important improvement over the NLO behaviour. In the same figure, we also show the effect of neglecting the contribution of Coulomb terms of order $O\left(\alpha_{s}^{4}\right)$ and higher. With the exception of the low-energy points, where Coulomb effects are very important because of the closeness of the threshold, inclusion of the Coulomb terms in the resummation is not a significant effect.

A more detailed representation of the scale sensitivity of the resummed top cross-section is shown in fig. 9, where we show the scale dependence in the wide range $m_{t} / 10<\mu<10 m_{t}$.

It was pointed out in ref. [7] that large NLL contributions to the resummation of leading logarithms arise from the inclusion of NLL corrections to the relation $1-z^{N} \sim \theta(1-z-1 / N)$, which can be used to perform the integrals in eqs. (39)-(42). This relation, which is valid at LL level, should be replaced at NLL order by $1-z^{N} \sim \theta\left(1-z-e^{-\gamma_{E}} / N\right)$. Neglecting terms beyond 


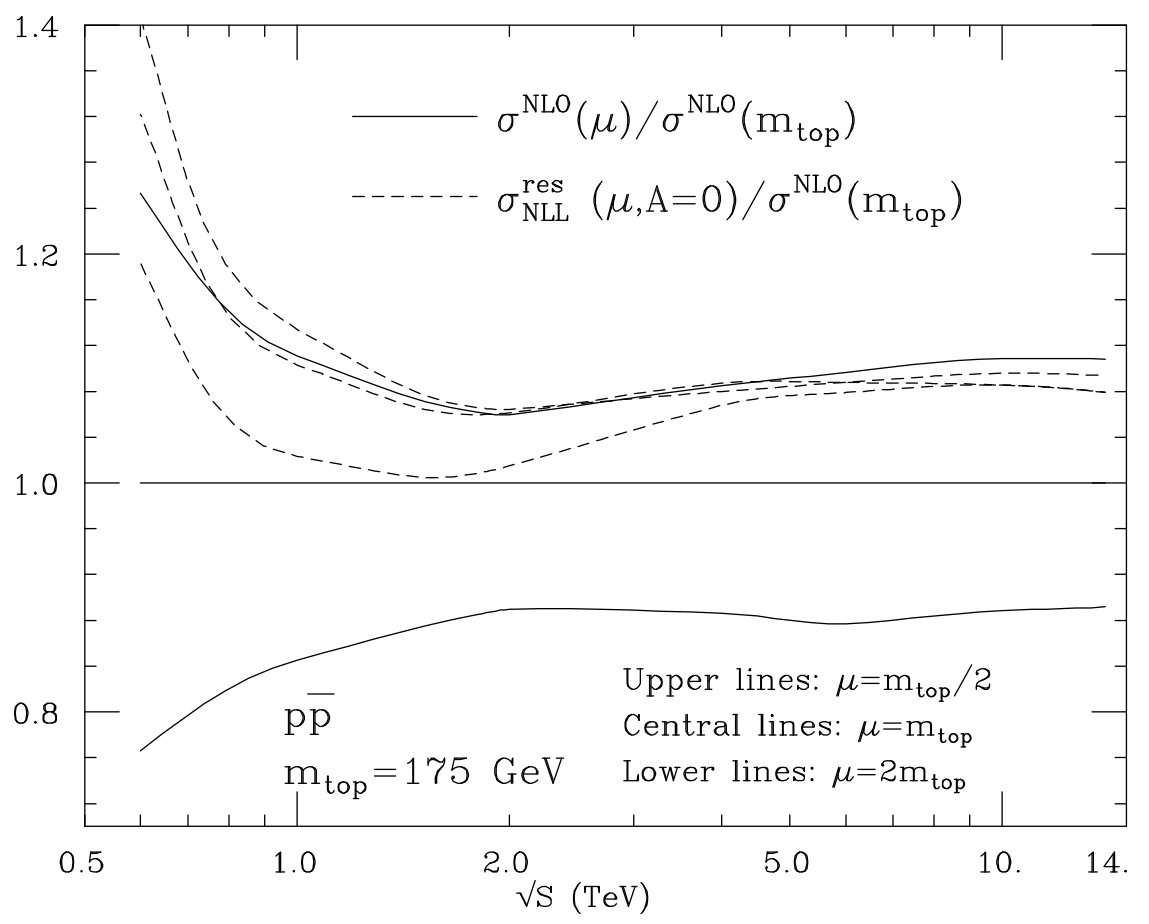

Figure 6: Scale-dependence of the $t \bar{t}$ production cross-section in $p \bar{p}$ collisions, as a function of $\sqrt{S}$. The solid lines represent the exact NLO result for different choices of $\mu$ $\left(\mu=m_{t} / 2\right.$ and $\left.2 m_{t}\right)$, normalised to the $\mu=m_{t}$ result. The solid lines represent the NLO+NLL result (with $A=0$ ) for different choices of $\mu\left(\mu=m_{t} / 2, m_{t}\right.$ and $\left.2 m_{t}\right)$, normalised to the NLO $\mu=m_{t}$ result.

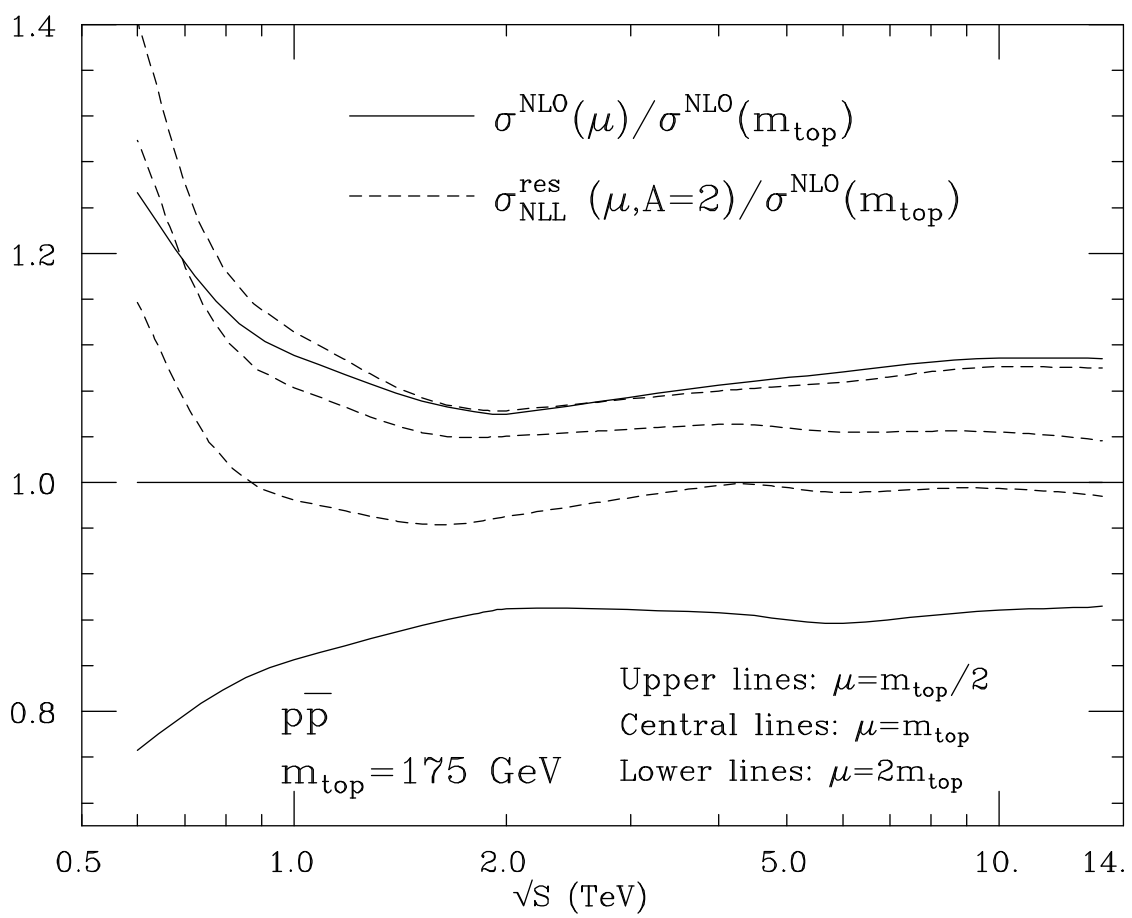

Figure 7: Same as fig. 6 , but for $A=2$. 


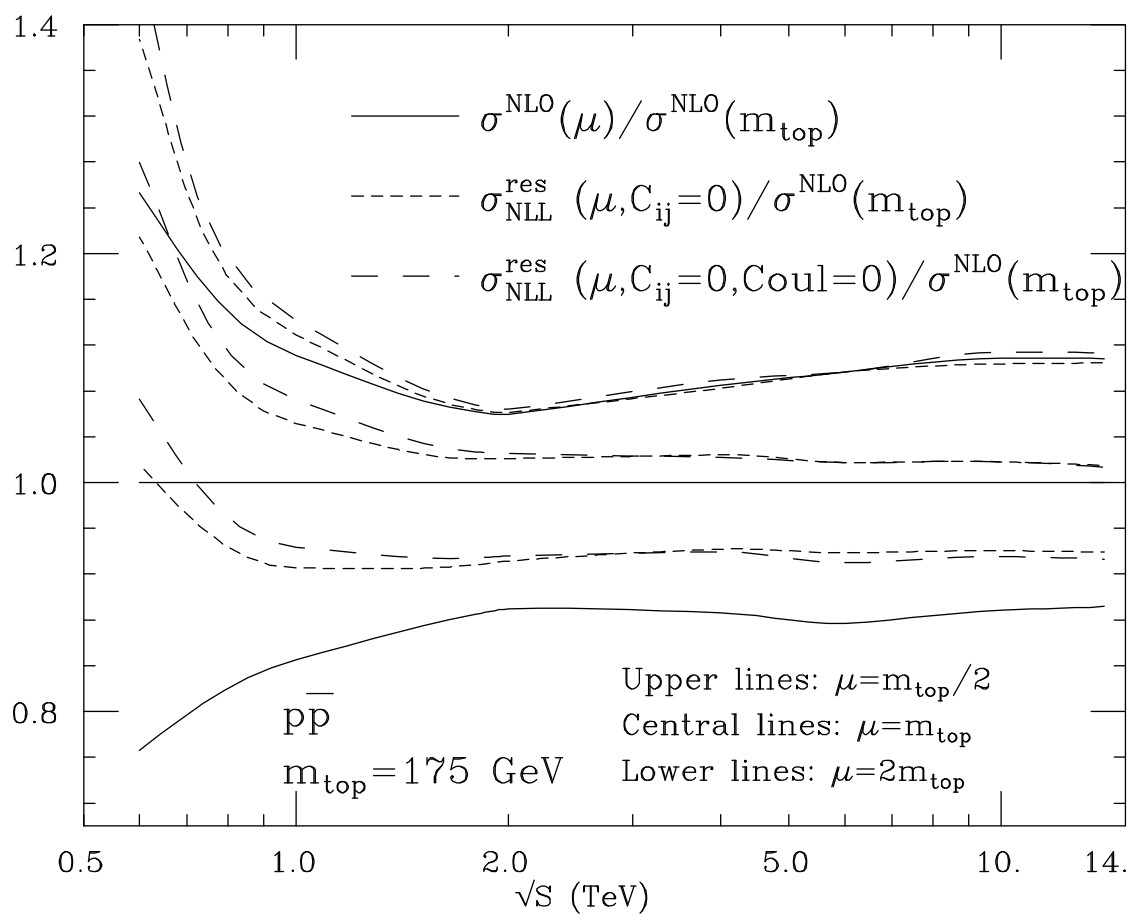

Figure 8: Same as fig. 6, but with the NNLL terms proportional to $C_{i j}$ set to zero (short-dashed lines). The dashed lines represent the same calculation, with the Coulomb contributions in eq. (55) removed.

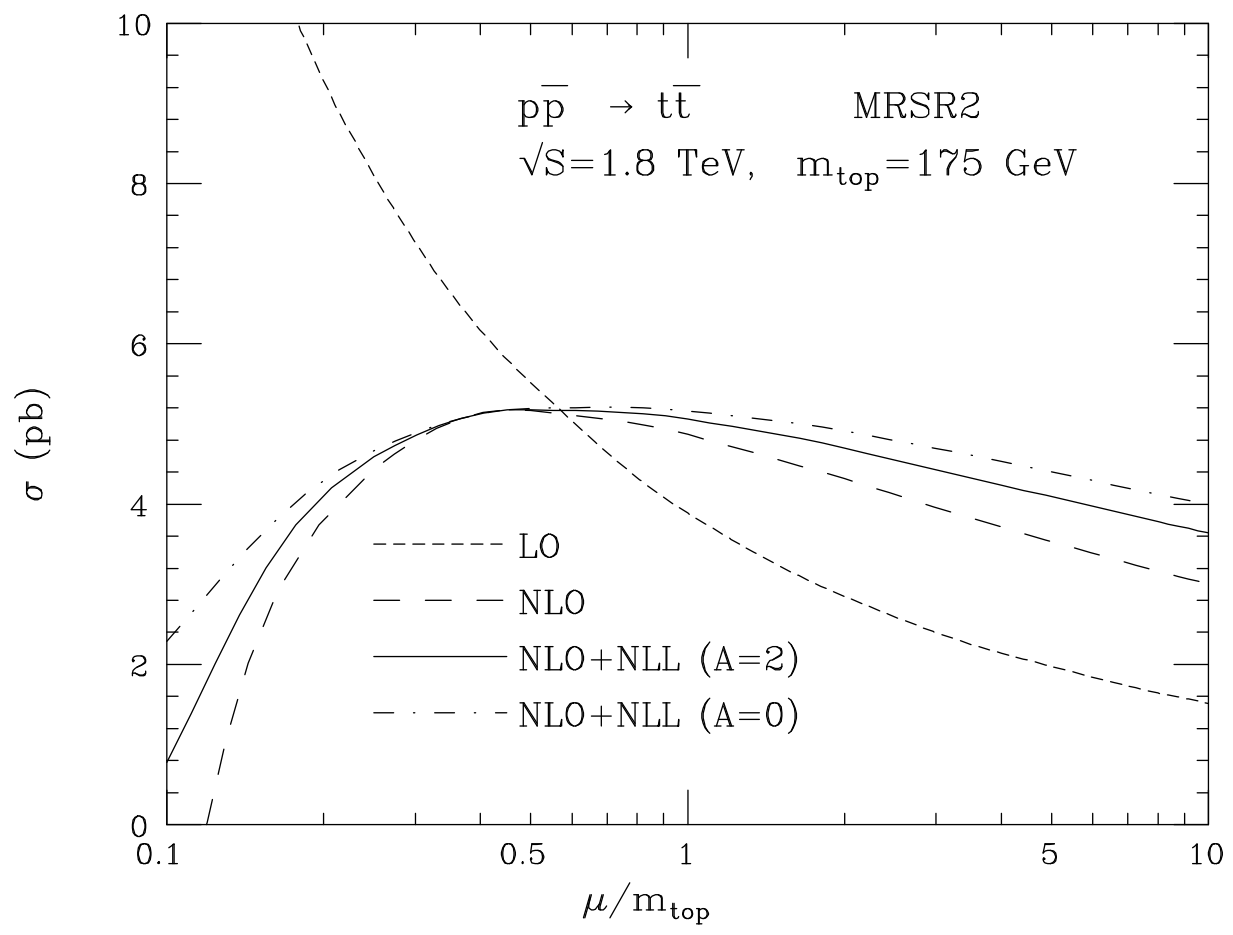

Figure 9: Scale dependence of the total $t \bar{t}$ production cross-section in $p \bar{p}$ collisions at $\sqrt{S}=1.8 \mathrm{TeV}$. LO (short-dashed line), NLO (dashed line) and NLO+NLL (solid and dot-dashed lines, for $A=2$ and $A=0$ respectively). 


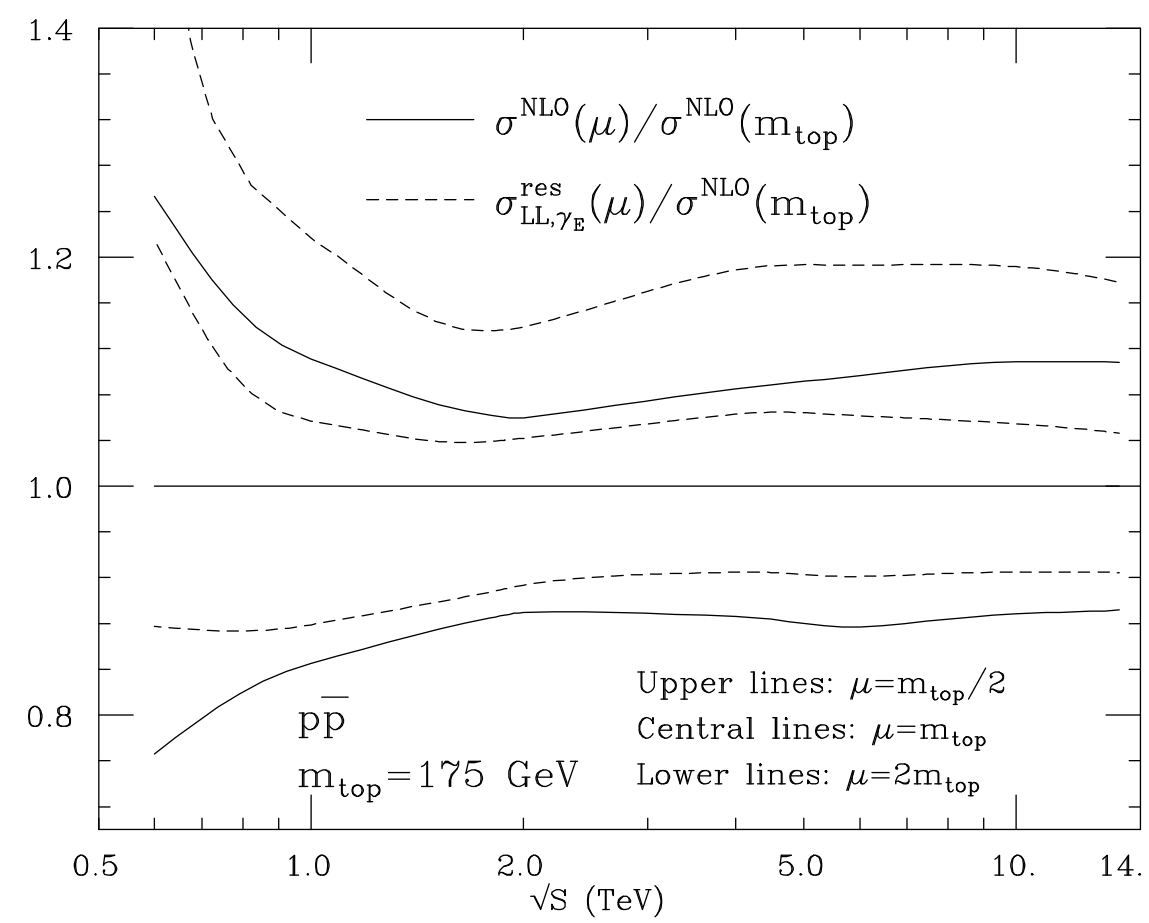

Figure 10: Same as fig. 6, but with the NLL result replaced with the resummation of the leading logarithms only, and inclusion of the $\gamma_{E}$ terms defined by eq. (65).

NLL accuracy, this replacement amounts to the substitution ${ }^{6}$ :

$$
\Delta_{i j, N}^{L L} \rightarrow\left(1+\gamma_{E} \frac{\partial}{\partial \ln N}\right) \Delta_{i j, N}^{L L}
$$

For the specific choice $\mu=m_{t}$, we showed in [7] that the $O\left(\alpha_{s}^{3}\right)$ truncation of the LL resummed calculation provides a better agreement with the exact NLO result for the partonic cross-section when this substitution is applied. This agreement is however accidental and limited to this particular choice of the renormalization scale. Figure 10 shows in fact that, with the sole substitution in eq. (65), no improvement in the scale dependence is observed relative to the NLO calculation when the scale is varied. Such an improvement can only be obtained when the full set of NLL terms is included.

The heavy-quark pair cross-section in $p \bar{p}$ collisions at $\sqrt{S}=1.8$ and $2 \mathrm{TeV}$ are shown, as a function of the heavy-quark mass, in figs. 11 and 12. We chose the value $A=2$, which gives a more conservative estimate of the scale uncertainty, and PDF set MRSR2 $\left(\alpha_{s}\left(M_{\mathrm{Z}}\right)=0.119\right)$. The two dashed lines correspond to the NLO result, with $\mu=m_{t} / 2$ and $2 m_{t}$. The two solid lines correspond to the fully resummed NLL result. At $\sqrt{S}=1.8 \mathrm{TeV}$ and for $m_{t}=175 \mathrm{GeV}$, we get a fully resummed result of $\sigma_{t \bar{t}}=5.06_{-0.36}^{+0.13} \mathrm{pb}$, compared to the fixed NLO one of $\sigma_{t \bar{t}}=4.87_{-0.56}^{+0.30} \mathrm{pb}$ (the central values representing the results with $\mu=m_{t}$ ). The scale uncertainty of the resummed result is reduced by almost a factor of 2 . The current experimental results from CDF [22] and D0 [23] are respectively: $\sigma_{t \bar{t}}=7.6_{-1.5}^{+1.8} \mathrm{pb}\left(\mathrm{CDF}\right.$, at $\left.m_{t}=175 \mathrm{GeV}\right)$, and $\sigma_{t \bar{t}}=5.5 \pm 1.8 \mathrm{pb}$ (D0, at $\left.m_{t}=173.3 \mathrm{GeV}\right)$.

\footnotetext{
${ }^{6}$ This is also equivalent to adding to $\ln \Delta_{i j}^{L} L$ the sole contributions proportional to $\gamma_{E}$ in the expressions (49) for $g_{i j}^{(2)}$.
} 


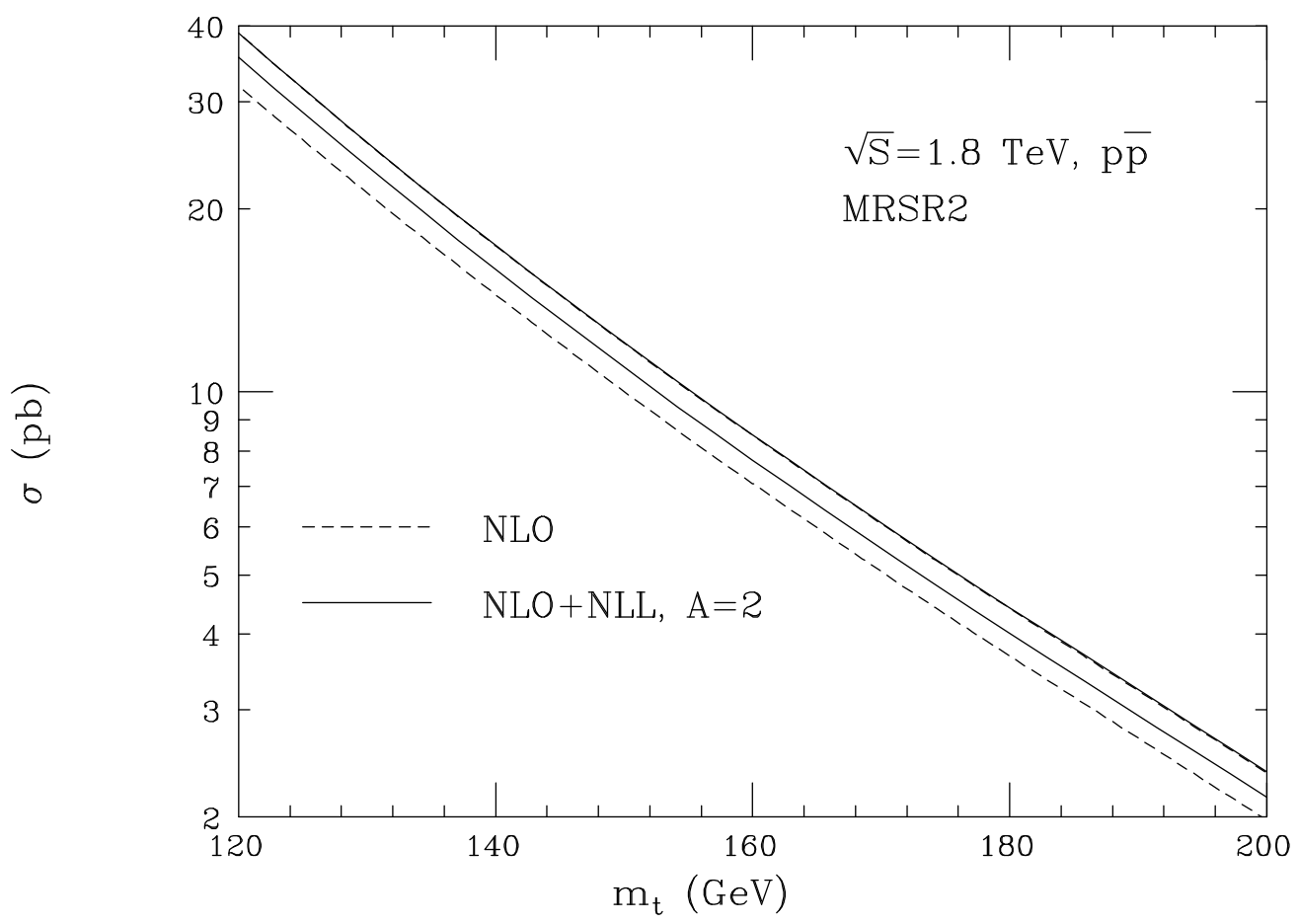

Figure 11: Total $t \bar{t}$ production cross-section in $p \bar{p}$ collisions at $\sqrt{S}=1.8 \mathrm{TeV}$, as a function of the top-quark mass. Dashed lines: NLO result; solid lines: NLO+NLL result. Upper lines: $\mu=m_{t} / 2$; lower lines: $\mu=2 m_{t}$.

The NLO and fully resummed cross-sections for LHC ( $p p$ collisions at $\sqrt{S}=14 \mathrm{TeV}$ ) are shown as a function of the heavy-quark mass in fig. 13.

In Table 1 we collect the values of the cross-sections for $m_{t}=175 \mathrm{GeV}$ at the Tevatron and LHC. The rapid convergence of the higher-order corrections is displayed in table 2, for the case of top production at the Tevatron. The sum of all entries in each row corresponds to the minimal prescription, while each fixed order term does not have any ambiguity due to the choice of the contour for the Mellin transformation in eq. (62). This supports the validity of the minimal prescription, since the truncated resummed expansion converges to it very rapidly.

Potentially large resummation effects should be expected in $b \bar{b}$ production at fixed target energies, due to the closeness of the threshold. As an example, we collect in Table 3 the values of the $b \bar{b}$ total cross-section calculated using the MRSR1 set $\left(\alpha_{s}\left(M_{\mathrm{Z}}\right)=0.112\right)$ for $p p$ collisions at $\sqrt{S}=39.2 \mathrm{GeV}$, the configuration of the upcoming HERAB experiment at DESY. We show both the NLO results and the fully resummed ones, for several values of the bottom quark mass and for the three standard choices of renormalization scale. The resummation corrections are large and positive for large values of the renormalization scale, but become small - and eventually negative for the smaller values of the renormalization scale. As a result, the overall scale dependence of the cross-section is significantly reduced in the fully resummed calculation, and the overall uncertainty band is fully included in the uncertainty band of the NLO calculation.

The NLO cross-section, and the resummation effects, are largely enhanced by the choice of the MRSR2 set, for which the value of the coupling constant is larger. The results are shown in Table 4. The conclusions regarding the improved stability of the resummed result, as well as the reliability of the uncertainty estimate obtained from the scale variation of the NLO calculation, 


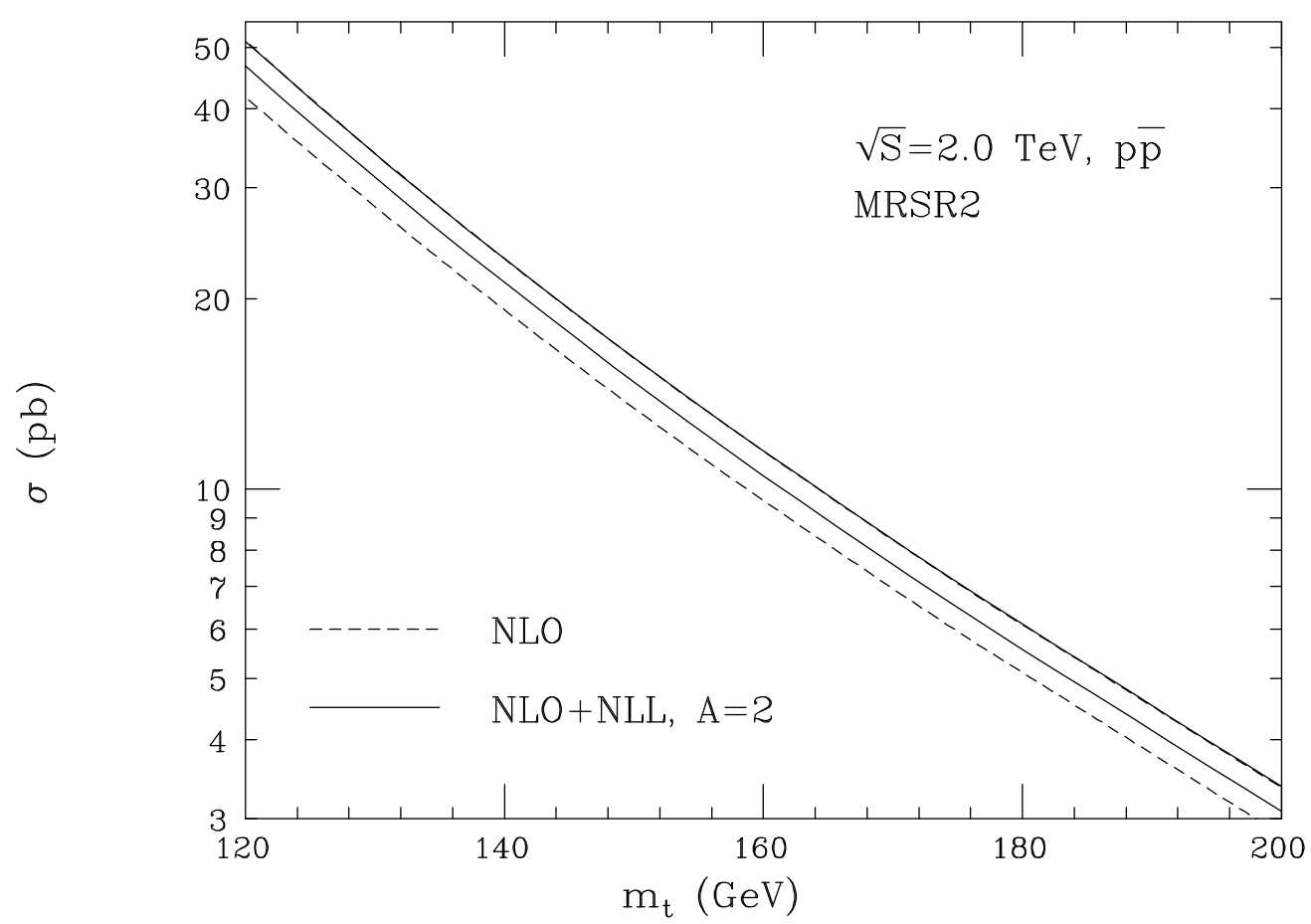

Figure 12: Total $t \bar{t}$ production cross-section in $p \bar{p}$ collisions at $\sqrt{S}=2.0 \mathrm{TeV}$, as a function of the top-quark mass. Dashed lines: NLO result; solid lines: NLO+NLL result. Upper lines: $\mu=m_{t} / 2$; lower lines: $\mu=2 m_{t}$.

\begin{tabular}{|c|cc|cc|cc|}
\hline & \multicolumn{2}{|c|}{$p \bar{p}$ at $\sqrt{S}=1.8 \mathrm{TeV}$} & \multicolumn{2}{|c|}{$p \bar{p}$ at $\sqrt{S}=2 \mathrm{TeV}$} & \multicolumn{2}{|c|}{$p$ at $\sqrt{S}=14 \mathrm{TeV}$} \\
\cline { 2 - 7 }$\mu_{\mathrm{R}}=\mu_{\mathrm{F}}$ & NLO & NLO+NLL & NLO & NLO+NLL & NLO & NLO+NLL \\
\hline \hline$m_{t} / 2$ & 5.17 & 5.19 & 7.10 & 7.12 & 893 & 885 \\
\hline$m_{t}$ & 4.87 & 5.06 & 6.70 & 6.97 & 803 & 833 \\
\hline $2 m_{t}$ & 4.31 & 4.70 & 5.96 & 6.50 & 714 & 794 \\
\hline
\end{tabular}

Table 1: Total $t \bar{t}$ cross-sections $\left(m_{t}=175 \mathrm{GeV}\right)$ at the Tevatron and LHC, in pb. PDF set MRSR2. 


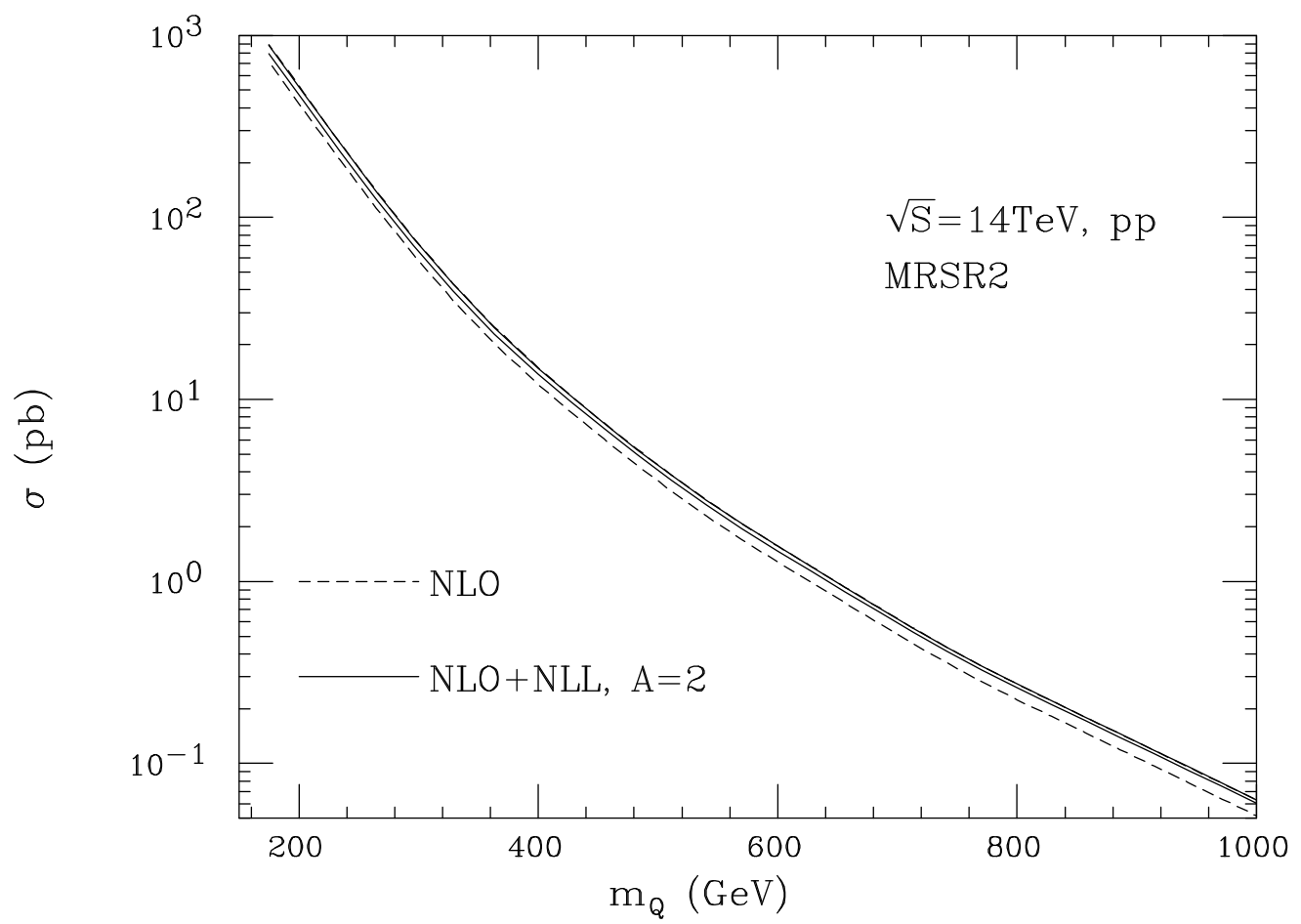

Figure 13: Total $Q \bar{Q}$ production cross-section in $p p$ collisions at $\sqrt{S}=14 \mathrm{TeV}$, as a function of the heavy-quark mass. Dashed lines: NLO result; solid lines: NLO+NLL result. Upper lines: $\mu=m_{Q} / 2$; lower lines: $\mu=2 m_{Q}$.

\begin{tabular}{|c|c|c|c|c|c|c|}
\hline & $\alpha_{s}^{2}$ & $\alpha_{s}^{3}$ & $\alpha_{s}^{4}$ & $\alpha_{s}^{5}$ & $\alpha_{s}^{6}$ & $\alpha_{s}^{\geq 7}$ \\
\hline \hline$q \bar{q}$ & 3590 & 766 & 60.2 & 2.4 & -0.6 & -0.08 \\
$g g$ & 298 & 264 & 98.1 & 26.1 & 5.8 & 1.5 \\
$q \bar{q}+g g$ & 3888 & 1030 & 158 & 28.5 & 5.1 & 1.4 \\
\hline
\end{tabular}

Table 2: Contributions to the total $t \bar{t}$ cross-sections (in $\mathrm{fb}$ ) at the Tevatron $(1.8 \mathrm{TeV})$ from higher orders in the expansion of the NLL resummed result, with $\mu=m_{t}, m_{t}=175$ $\mathrm{GeV}$ and PDF set MRSR2. The second column gives the exact NLO result. The last row only includes the sum of the $q \bar{q}+g g$ channels. 


\begin{tabular}{|c|cc|cc|cc|}
\hline \multirow{2}{*}{$\mu_{\mathrm{R}}=\mu_{\mathrm{F}}$} & \multicolumn{2}{|c|}{$m_{b}=4.5 \mathrm{GLO}$} & \multicolumn{2}{c|}{$m_{b}=4.75 \mathrm{GeV}$} & \multicolumn{2}{c|}{$m_{b}=5.0 \mathrm{GeV}$} \\
\cline { 2 - 7 } & $\mathrm{NLO}+\mathrm{NLL}$ & $\mathrm{NLO}$ & $\mathrm{NLO}+\mathrm{NLL}$ & $\mathrm{NLO}$ & NLO+NLL \\
\hline \hline$m_{b} / 2$ & 27.4 & 27.3 & 17.6 & 17.7 & 11.2 & 11.5 \\
\hline$m_{b}$ & 15.7 & 22.8 & 9.90 & 14.9 & 6.26 & 9.75 \\
\hline $2 m_{b}$ & 8.74 & 18.0 & 5.47 & 11.7 & 3.43 & 7.63 \\
\hline
\end{tabular}

Table 3: Total $b \bar{b}$ cross-sections (in nb) at HERAB ( $p p$ at $\sqrt{S}=39.2 \mathrm{GeV}$ ), as a function of the the $b$ mass $m_{b}$. PDF set MRSR1.

\begin{tabular}{|c|cc|cc|cc|}
\hline & \multicolumn{2}{|c|}{$m_{b}=4.5 \mathrm{GeV}$} & \multicolumn{2}{c|}{$m_{b}=4.75 \mathrm{GeV}$} & \multicolumn{2}{c|}{$m_{b}=5.0 \mathrm{GeV}$} \\
\cline { 2 - 7 }$\mu_{\mathrm{R}}=\mu_{\mathrm{F}}$ & $\mathrm{NLO}$ & $\mathrm{NLO}+\mathrm{NLL}$ & $\mathrm{NLO}$ & $\mathrm{NLO}+\mathrm{NLL}$ & $\mathrm{NLO}$ & NLO+NLL \\
\hline \hline$m_{b} / 2$ & 45.2 & 44.9 & 28.6 & 28.9 & 18.0 & 18.6 \\
\hline$m_{b}$ & 22.2 & 35.3 & 13.9 & 22.9 & 8.71 & 14.9 \\
\hline $2 m_{b}$ & 11.2 & 26.1 & 6.94 & 17.0 & 4.31 & 11.0 \\
\hline
\end{tabular}

Table 4: Total $b \bar{b}$ cross-sections (in nb) at HERAB $(p p$ at $\sqrt{S}=39.2 \mathrm{GeV})$, as a function of the the $b$ mass $m_{b}$. PDF set MRSR2. 


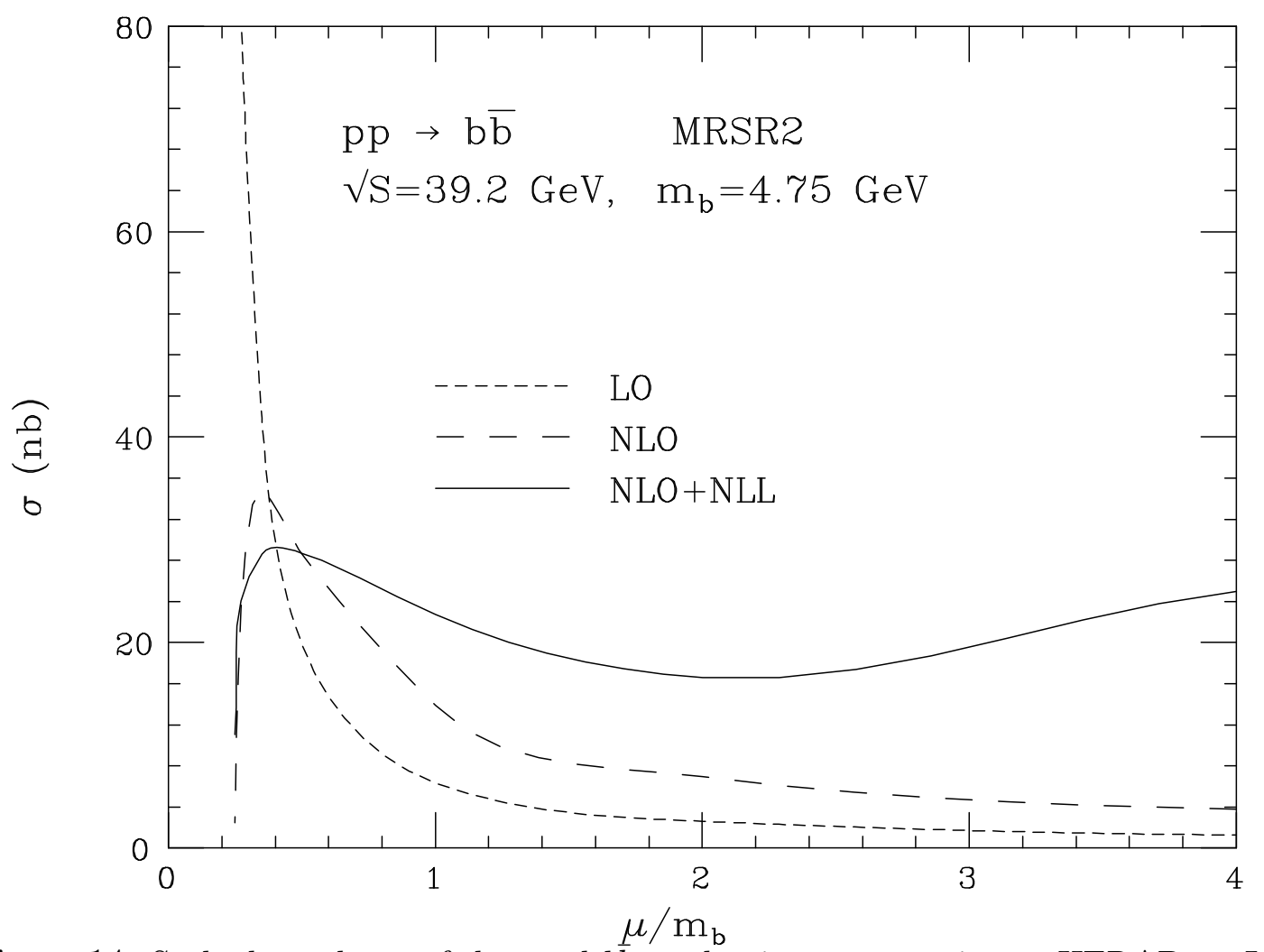

Figure 14: Scale dependence of the total $b b$ production cross-section at HERAB, at LO (dotted line), NLO (dashed line) and NLO+NLL (solid line).

are nevertheless unchanged. A plot of the scale dependence of the LO, NLO and resummed cross-sections, for $m_{b}=4.75 \mathrm{GeV}$ and PDF set MRSR2 is shown in fig. 14 .

As a comparison, we give here the value of the bottom-pair cross-section at the Tevatron, where we expect much smaller resummation effects. The NLO cross-section at the Tevatron $\left(m_{b}=\right.$ $4.75 \mathrm{GeV}$, MRSR2) is: $\sigma=74.8,56.2,46.6 \mu \mathrm{b}$ for $\mu=m_{b} / 2, m_{b}, 2 m_{b}$. Including resummation we obtain: $\sigma=74.2,57.6,52.2 \mu \mathrm{b}$ for $\mu=m_{b} / 2, m_{b}, 2 m_{b}$. Some reduction in the scale dependence can be seen, but not at the level exhibited by the HERAB results. This is consistent with the fact that $b$ production at the Tevatron takes place far away form the threshold, and the class of logarithms resummed in this work cannot be expected to give a dominant contribution.

To conclude, we show in fig. 15 the effect of resummation on the total cross-section for fixedtarget hadroproduction of charm-quark pairs. The typical values of $4 \mathrm{~m}_{c}^{2} / S$ at fixed-target energies (where $\sqrt{S}$ is in the range $20-40 \mathrm{GeV}$ ) are close to the values for top-pair production at the Tevatron. Contrary to the top case, however, charm production is dominated in $p p$ collisions by the $g g$ annihilation channel, due to the smallness of the antiquark sea. Considering in addition the larger size of the strong coupling constant at scales of the order of the charm mass, we therefore expect the resummation corrections to be large. Results are plotted as a function of the beam energy, for proton-induced reactions. The interval for the scale variation is limited to the range $m_{c}<\mu<2 m_{c}$, since the parton densities we use (MRSR2) are limited to the domain $\mu^{2}>1.25 \mathrm{GeV}^{2}$. Once more, the resummed result shows a significant reduction in the scale dependence over the NLO result. 


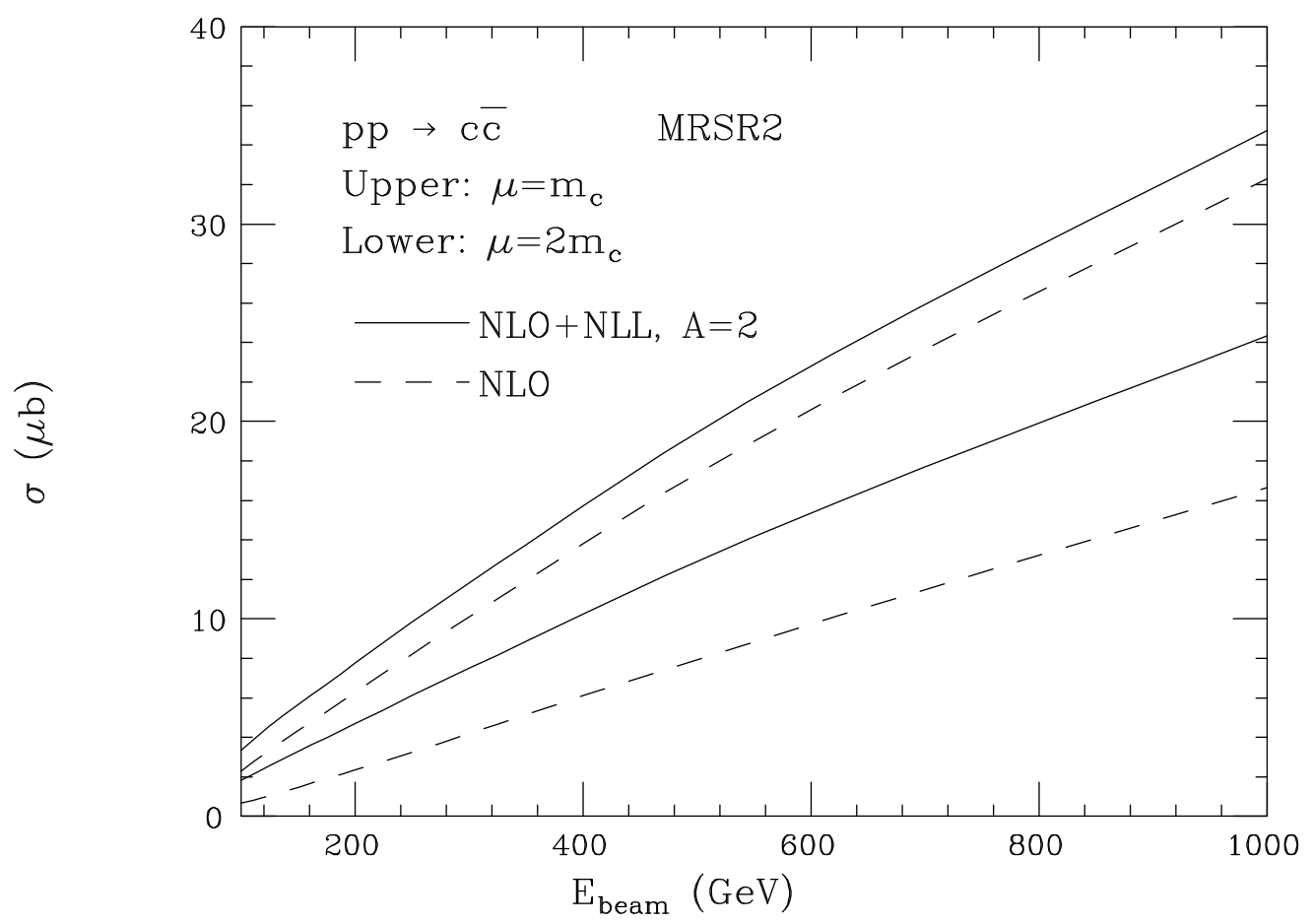

Figure 15: Total $c \bar{c}$ cross-section in fixed-target $p p$ collisions, as a function of beam energy, at the NLO and NLO+NLL.

\section{Discussion and conclusions}

We presented in this paper the first calculation of the heavy-quark hadro-production total crosssection accurate up to, and including, next-to-leading threshold-enhanced logarithms, resummed at all orders of perturbation theory. One can show that our analytical results for the resummed total cross-section are consistent with those obtained in in [8] for the cross-section at fixed invariant mass of the heavy-quark pair. When the resummed partonic cross-sections are convoluted with hadronic parton densities, a significant improvement is observed in the stability of the results with respect to changes of the renormalization scale. In the case of top-quark production at the Tevatron, the corrections relative to the NLO calculation are of the order of $9 \%$ for $\mu=2 m_{t}$, $4 \%$ for $\mu=m_{t}$, and $0.5 \%$ for $\mu=m_{t} / 2$. The result for $\mu=m_{t}$ can be summarized as follows. Including LL terms we get a $0.5 \%$ correction; the correction increases to $4 \%$ when higher-order terms proportional to $\gamma_{E}$ are included; when all NLL contributions are included the correction decreases to 2.6\%; when the Coulomb term is added the correction decreases to $2.1 \%$; when the first NNLL terms (the $C_{i j}$ constants) are included the correction grows to $4 \%$ for the choice $A=2$, to $6 \%$ for the choice $A=0$. As we have seen, the $A=0$ choice is somewhat disfavoured, since it overestimates the $O\left(\alpha_{s}^{3}\right)$ cross-section above threshold. We emphasize that the overall compression of the uncertainty band toward the high cross-section values is significant and always present once we include the NLL terms, regardless of the presence of the $C_{i j}$ and Coulomb terms, and regardless of our choice of the value of $A$.

In the case of top productio at the Tevatron, it was shown in ref. [7] that inclusion of the sole NLL terms proportional to $\gamma_{E}$ provided an improvement in the agreement with the $O\left(\alpha_{s}^{3}\right)$ partonic cross-section for the choice of scale $\mu=m_{t}$. This improvement is however accidental, and indeed, as shown in this paper, it is not sufficient to improve the accuracy of the resummed 
calculation when the renormalization scale is changed to different values. Only at the full NLL level a significant reduction in the scale dependence is found. Similar results are obtained at higher energies, including the energy of the future LHC collider.

The improvement in the predictive power allowed by the calculation we presented in this work is even more impressive when one considers the case of the bottom-quark production near threshold, at the future HERAB experiment. In this case the size of the strong coupling constant gives rise to very large perturbative corrections, which result in a factor of 4 uncertainty in the NLO result at a fixed value of the bottom mass and parton densities. Inclusion of resummation effects stabilizes the predicted cross-section at the level of $\pm 50 \%$. As in the case of the top cross-sections, the evaluation of the fully resummed cross-sections shows that the estimates of the theoretical uncertainty at the NLO obtained by varying the renormalization scales in the range $m_{Q} / 2<\mu<2 m_{Q}$ were correct. While the resummed result has a much smaller uncertainty than the fixed-order one, the resulting range of predictions is always included in the uncertainty band estimated at the NLO.

Acknowledgement: We thank Matteo Cacciari for pointing out an error in Fig. 14 of the first version of this paper.

\section{References}

[1] G. Sterman, Nucl. Phys. B281 (1987) 310.

[2] S. Catani and L. Trentadue, Nucl. Phys. B327 (1989) 323, Nucl. Phys. B353 (1991) 183.

[3] E. Laenen, J. Smith and W.L. van Neerven, Nucl. Phys. B369 (1992) 543.

[4] E. Laenen, J. Smith and W.L. van Neerven, Phys. Lett. B321 (1994) 254.

[5] E. Berger and H. Contopanagos, Phys. Lett. B361 (1995) 115, Phys. Rev. D54 (1996) 3085, ibid. 57 (1998) 253.

[6] S. Catani, M.L. Mangano, P. Nason and L. Trentadue, Phys. Lett. B351 (1996) 555.

[7] S. Catani, M.L. Mangano, P. Nason and L. Trentadue, Nucl. Phys. B478 (1996) 273.

[8] N. Kidonakis, PhD thesis (SUNY, Stony Brook), hep-ph/9606474;

N. Kidonakis and G. Sterman, Phys. Lett. 387 (1996) 867, Nucl. Phys. B505 (1997) 321.

[9] R. Bonciani, University of Florence Thesis, July 1997 (in Italian).

[10] N. Kidonakis, G. Oderda and G. Sterman, EDINBURGH-97-22, hep-ph/9801268.

[11] N. Kidonakis, J. Smith and R. Vogt, Phys. Rev. D56 (1997) 1553.

[12] R. Bonciani, S. Catani, M.L. Mangano and P. Nason, in preparation.

[13] P. Nason, S. Dawson and R. K. Ellis, Nucl. Phys. B303 (1988) 607.

[14] W. Beenakker, H. Kuijf, W.L. van Neerven and J. Smith, Phys. Rev. D40 (1989) 54.

[15] J. Kodaira and L. Trentadue, Phys. Lett. B112 (1982) 66.

[16] S. Catani, E. d'Emilio and L. Trentadue, Phys. Lett. B211 (1988) 335.

[17] S. Catani, G. Marchesini and B.R. Webber, Nucl. Phys. B349 (1991) 635.

[18] M. Krämer, E. Laenen and M. Spira, preprint CERN-TH/96-231, hep-ph/9611272.

[19] S. Catani, L. Trentadue, G. Turnock and B.R. Webber, Nucl. Phys. B407 (1993) 3.

[20] R.K. Ellis and D.A. Ross, Nucl. Phys. B345 (1990) 79;

S. Catani, M. Ciafaloni and F. Hautmann, Phys. Lett. B242 (1990) 97, Nucl. Phys. B366 (1991) 135;

J.C. Collins and R.K. Ellis, Nucl. Phys. B360 (1991) 3.

[21] A.D. Martin, R.G. Roberts and W.J. Stirling, Phys. Lett. B387 (1996) 419 
[22] F. Abe et al., CDF Collaboration, FERMILAB-PUB-97/286-E.

[23] S. Abachi et al., D0 Collaboration, Phys. Rev. Lett. 79 (1997) 1203. 